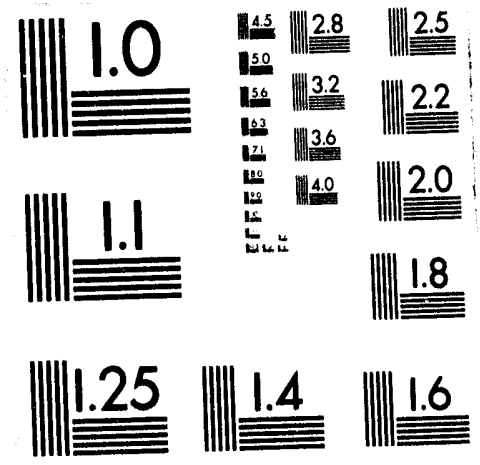



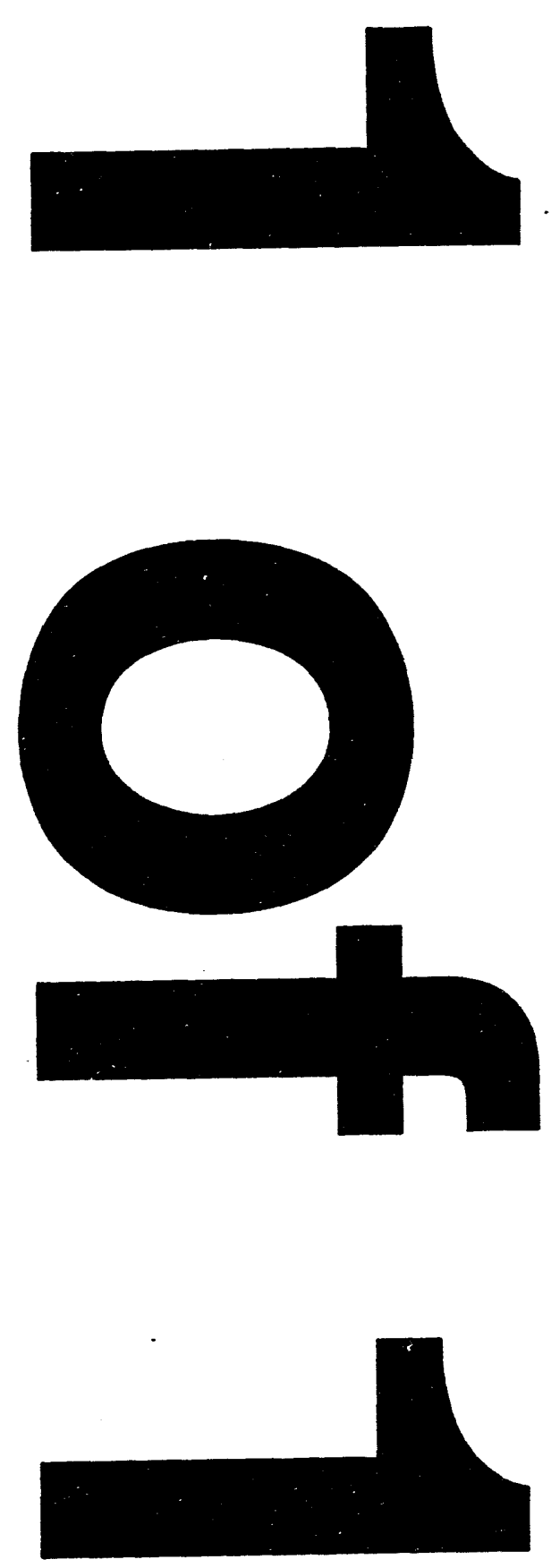


\section{SOLUTION OF MATHEMATICAL PROGRAMMING FORMULATIONS OF SUBGAME PERFECT EQUILIBRIUM PROBLEMS

Charles M. Macal

Environmental Assessment and Information Sciences Division

Argonne National Laboratory

Argonne, IL 60439

Arthur P. Hurter

Department of Industrial Engineering and Management Sciences

Northwestem University

Evanston, IL 60208

\section{DISCLAIMER}

This report was prepared as an account of work sponsored by an agency of the United States Government. Neither the United States Government nor any agency thereof, nor any of their employees, makes any warranty, express or implied, or assumes any legal liability or responsibility for the accuracy, completeness, or usefulness of any information, apparatus, product, or process disclosed, or represents that its use would not infringe privately owned rights. Reference herein to any specific commercial product, process, or service by trade name, trademark, manufacturer, or otherwise does not necessarily constitute or imply its endorsement, recommendation, or favoring by the United States Government or any agency thereof. The views and opinions of authors expressed herein do not necessarily state or reflect those of the United States Government or any agency thereof.

February 12, 1992 


\title{
SOLUTION OF MATHEMATICAL PROGRAMMING FORMULATIONS \\ OF SUBGAME PERFECT EQUILIBRIUM PROBLEMS
}

\author{
Charles M. Macal and Arthur P. Hurter
}

\begin{abstract}
Mathematical programming models have been developed to represent imperfectly competitive (oligopolistic) market structures and the interdependencies of decision-making units in establishing prices and production levels. The solution of these models represents an economic equilibrium. A subgame perfect equilibrium formulation explicitly considers that each agent's strategies depend on the current state of the system; the state depends solely on previous decisions made by the economic agents. The structure of an industry-wide model that is formulated as a subgame perfect equilibrium problem is a matrix of simultaneous mathematical programming problems, where the rows represent time periods and the columns represent agents. This paper formally defines the subgame perfect equilibrium problem that includes mathematical programs for agent decision problems, and it characterizes the feasible space in a way that is conducive to the solution of the problem. The existence of equilibrium solutions on convex subspaces of the feasible region is proved, and this set is shown to contain the subgame perfect equilibrium solutions. A procedure for computing equilibrium solutions and systematically searching the subspaces is illustrated by a numerical example.
\end{abstract}

Keywords: Games/group decisions, Noncooperative: formulation of subgame perfect equilibria Economics: competitive market models, economic equilibria 


\title{
SOLUTION OF MATHEMATICAL PROGRAMMING FORMULATIONS \\ OF SUBGAMI PERFECT EQUILIBRIUM PROBLEMS
}

\author{
Charles M. Macal and Arthur P. Hurter
}

Mathematical programming models have been developed to represent industries and markets at an ever increasing level of detail and sophistication. They are used by the U.S. Department of Energy and other govemmental and private-sector organizations in making strategic policy decisions. Realistic representations of decision making in these models include resource constraints, such as available financial and physical resources, capacities of production facilities, and restrictions of physical units to non-negative values. These models also consider the imperfectly competitive nature of the markets and the interdependencies of decision-making units in establishing prices and production levels. The market representation is an oligopoly, and the solution of the models represents an economic equilibrium in the sense described below. Because there are a number of theories about how oligopoly prices and production come about and how agents interact, numerous equilibrium concepts have been adapted from the field of game theory. Selecting an equilibrium concept that is appropriate for modeling a particular market is a central problem in formulating an imperfect competition model.

The Nash equilibrium concept (Nash 1951) from noncooperative game theory is commonly applied to oligopolistic market models. Static Nash equilibrium models assume that each agent recognizes that its actions affect the market, but an agent takes the actions of other agents as constant, or fixed, when making decisions to maximize utility. Equilibrium is a condition in which no agent can unilaterally increase utility by adopting any strategy other than its equilibrium strategy, given that other agents adopt their equilibrium strategies. Dynamic Nash equilibrium formulations explicitly consider that each agent's strategies depend on the current state of the system which, in the deterministic case, depends solely on the decisions previously made by all agents. For rational agents, it is reasonable to model agents' strategies as being optimal with respect to the current state. 
Selten $(1965,1973,1975)$ introduced the notion of subgame perfect equilibrium (SPE) for games in extensive form, but this concept has direct applicability to imperfectly competitive markets as well. An SPE is a set of strategies that induces an equilibrium on every subgame of the game. In an oligopoly, a subgame is a decision point and is distinguished by an agent and a time period; an SPE is a set of strategies for all agents, where each strategy indicates the optimal decision of an agent for every time period as it depends on the state of the system at the time the decision is made.

Macal and Hurter (1991) formulated a subgame perfect equilibrium problem as a system of mathematical programming problems. An SPE problem can be thought of as a matrix of simultaneous mathematical programming problems, where the rows represent time periods and the columns represent agents. This paper describes a solution algorithm for SPE problems formulated as such a system of mathematical programs. A small problem is addressed consisting of two agents and two time periods, but application of the algorithm to arbitrarily large problems is straightforward.

This paper is organized as follows. Section 1 defines the SPE problem and characterizes the feasible space in a way that is conducive to its solution. Section 2 proves the existence of facet equilibrium solutions, the set of which contains the SPE solutions. Section 3 describes a procedure for computing facet equilibrium solutions and systematically searching facets for an SPE solution.

\section{SUBGAME PERFECT EQUILIBRIUM FORMULATION}

This section formulates the general subgame perfect equilibrium problem, presents an example for two agents and two time periods, and characterizes the feasible space in a way that is conducive to the solution of the SPE problem. 


\subsection{Notation}

$\alpha$

$\tau$

$x_{i t} \in \boldsymbol{R}^{n}$

$x_{t}=\left(x_{1 t}, \ldots, x_{i t}, \ldots, x_{\alpha t}\right)$

$x_{-i t}=\left(x_{1,}, \ldots, x_{i-1, l}, x_{i+1, t}, \ldots, x_{\alpha t}\right)$

$x_{i t}^{*} \in \boldsymbol{R}^{n}$

$X_{i k}\left(x_{l}, \ldots, x_{t-1}\right)$

$X_{t}=\left(X_{l t}, \ldots, X_{i t}, \ldots, X_{\alpha t}\right)$

$u_{i n}\left(x_{1}, \ldots, x_{t}\right)$

$\beta_{i t}$

$U_{i}\left(x_{1}, \ldots, x_{v}\right)=\Sigma_{t=l, \tau} \beta_{i t} u_{i t}$

$G_{i r}\left(x_{1}, \ldots, x_{t}\right)$

$z(x)=\operatorname{SolMax} g(x, y)$ number of economic agents or firms, assumed to be finite.

number of time periods, assumed to be finite.

decision variable (production) of agent (firm) $i$ at time $t$.

production by all firms at $t$.

production by firms other than $i$ at $t$.

optimal production of firm $i$ at $t$.

strategy mapping of all decisions preceding $t$ (history at $t$ ) to a

decision by agent $i$ at $t$.

strategies of all firms at $t$.

utility function of $i$ at $t$ assumed to depend on all decisions up to and including $\mathrm{t}$.

discount factor for firm $i$ at time $t$.

utility function of $i$, assumed to be separable.

constraint set for agent $i$ at time $t$, assumed to be linear.

$z$ is the solution (assumed to be unique) of $\operatorname{Max}_{y} g(x, y)$ as $x$ varies.

$y_{n}^{*}=\operatorname{Sol}_{n} \operatorname{Max}_{y_{1}, \ldots, y_{a}, \ldots} u\left(y_{1}, \ldots, y_{n}, \ldots\right) \quad \dot{y}_{n}^{*}$ is the $n^{\text {hn }}$ solution variable of $\operatorname{Max}_{\mathrm{y}} \mathrm{u}(\mathrm{y})$.

$\left(x_{1}^{*}, \ldots, x_{\alpha}^{*}\right)=\operatorname{Esol}_{i=1}^{\alpha}\left[\operatorname{Max}_{x_{i}} u_{i}\left(x_{i}, x_{-i}\right)\right] \quad\left(x_{1}^{*}, \ldots, x_{i}^{*}, \ldots, x_{\alpha}^{*}\right)$ is the solution to the equilibrium problem in brackets for agents 1 through $\alpha$, that is $x_{i}^{*}=$ Sol $\operatorname{Max}_{x} u_{i}\left(x_{i}, x^{*}\right)$ for $i=1, \ldots, \alpha$. 


\subsection{General Formulation}

Definition 1: A subgame perfect equilibrium (SPE) outcome $\left(x_{1}^{*}, \ldots, x_{v}^{*}\right)$ is a solution to the following system of optimization problems where $x_{1}^{*}$ solves at $t=1$ :

$\left(x_{11}^{*}, \ldots, x_{i 1}^{*}, \ldots, x_{\alpha 1}^{*}\right)=\operatorname{Esol}_{i=1}^{\alpha}\left[\begin{array}{cl}\operatorname{Max}_{x_{i 1} 20}: & u_{i 1}\left(x_{i 1}, x_{-i 1}\right)+\sum_{s=2}^{\tau} \beta_{i s} u_{i s}\left(x_{i 1}, x_{-i 1}, X_{2}, \ldots, X_{s}\right) \\ \text { S.t. }: & G_{i 1}\left(x_{i 1}, x_{-i 1}\right) \geq 0\end{array}\right]$

where for $t>1$ and $t<\tau(\tau>2)$, the strategies $X_{t}$ in $(1 a)$ are recursively defined as parameterized solutions to the following equilibrium system:

$X_{t}\left(x_{1}, \ldots, x_{t-1}\right)=$

$$
E s o l_{i=1}^{\alpha}\left[\begin{array}{cl}
\operatorname{Max}_{x_{i 2} \geq 0}: & u_{i t}\left(x_{1}, \ldots, x_{t-1} ; x_{i t}, x_{-i t}\right)+\sum_{s=t+1}^{\tau} \beta_{i s} u_{i s}\left(x_{1}, \ldots, x_{t-1} ; x_{i t}, x_{-i t}, X_{t+1}, \ldots, X_{s}\right) \\
\text { S.t. }: & G_{i t}\left(x_{1}, \ldots, x_{t-1} ; x_{i t}, x_{-i t}\right) \geq 0
\end{array}\right]
$$

with $x_{t}^{*}=X_{t}\left(x_{1}^{*}, \ldots, x_{t-1}^{*}\right)$

and for $t=\tau$, the strategies $X_{\tau}$ are defined as parameterized solutions to the following equilibrium system:

$X_{\tau}\left(x_{1}, \ldots, x_{\tau-1}\right)=\operatorname{Esol}_{i=1}^{\alpha}\left[\begin{array}{rl}\operatorname{Max}_{x_{n} \geq 0}: & u_{i \tau}\left(x_{1}, \ldots, x_{\tau-1} ; x_{i \tau}, x_{-i \tau}\right) \\ \text { S.t. : } & G_{i \tau}\left(x_{1}, \ldots, x_{\tau-1} ; x_{i \tau}, x_{-i \tau}\right) \geq 0\end{array}\right]$

with $x_{\tau}^{*}=X_{\tau}\left(x_{1}^{*}, \ldots, x_{\tau-1}^{*}\right)$

A subgame perfect equilibrium problem is a system of mathematical programming problems, as illustrated in Figure 1 (Macal and Hurter, 1991). This system has structural characteristics of both (static) oligopoly equilibrium systems (Murphy et al., 1982) and multilevel mathematical programs (Bard and Falk, 1982). 
The mapping $\mathrm{X}_{\mathrm{it}}\left(\mathrm{x}_{1}, \ldots \mathrm{x}_{\mathrm{t}-1}\right)$ is the equilibrium strategy for agent $\mathrm{i}$ at time $\mathrm{t}$ given the history $\left\{\mathrm{x}_{1}\right.$, $\left.\ldots, \mathrm{x}_{\mathrm{t}-1}\right\}$ of decisions preceding t. $\mathrm{X}_{\mathrm{it}}$ is called a strategy because it specifies an optimal equilibrium decision in period $t$ for any decisions $\left\{x_{1}, \ldots, x_{t-1}\right\}$ made preceding period $t$. Conceptually, SPE strategies can be constructed by a process of backward recursion, as in dynamic programming. The equilibrium strategies for every possible state of the game (market) in the last period are constructed first; the equilibrium strategies for every possible state of the game in the next-to-last period are constructed next, based on the observation that only equilibrium strategies for the last period need to be considered in computing equilibrium strategies for the next-to-last period, and so on.

An equilibrium in which strategies depend only on time (that is, strategies are not functions of the state of the system) is known as an open-loop equilibrium. For example, in an open-loop model, each agent takes the decisions of the other agents for all time periods as fixed and chooses decisions that maximize discounted profit for all time periods. Typically, open-loop models are considerably easier to solve than dynamic equilibrium models, which have state-dependent strategies. The open-loop formulation is presented here because of its significance in computing SPE solutions.

Definition 2: An open-loop equilibrium (OLE) outcome $\left(x_{1}^{*}, \ldots, x_{\tau}^{*}\right)$ is a solution to the following system of optimization problems:

$$
\left(x_{1}^{*}, \ldots, x_{i}^{*}, \ldots, x_{\alpha}^{*}\right)=\operatorname{Esol}_{i=1}^{\alpha}\left[\begin{array}{cl}
\operatorname{Max}_{\left(x_{i 1}, \ldots, x_{i j}\right) \geq 0}: & \sum_{s=1}^{\tau} \beta_{i s} u_{i s}\left(x_{i 1}, x_{-i 1}^{*}, x_{i 2}, x_{-i 2}^{*}, \ldots, x_{i \tau}, x_{-i \tau}^{*}\right) \\
\text { S.t. }: & G_{i 1}\left(x_{i 1}, x_{-i 1}^{*}\right) \geq 0 \\
\ldots & \ldots \\
& G_{i \tau}\left(x_{i 1}, x_{-i 1}^{*}, \ldots, x_{i \tau}, x_{-i \tau}^{*}\right) \geq 0
\end{array}\right]
$$

An open-loop equilibrium is a set of scalars, whereas an SPE is a set of mappings. This paper addresses computing SPE outcomes, which are scalars that result if all SPE strategies are acted upon. 


\subsection{Two-Period, Two-Agent Formulation}

For the remainder of this paper we consider the SPE formulation for two agents ( $\alpha=2$, agents i and $\mathrm{k}$ ) and two time periods $(\tau=2)$. We also assume that all constraints $\mathrm{G}$ are linear and utility functions $U$ are quadratic. The period-two equilibrium problem from (1c) above is to find $\left(x^{*}, x^{*}{ }_{k 2}\right)$ for any $\left(\mathbf{x}_{\mathrm{i} 1}, \mathbf{x}_{\mathbf{k} 1}\right)$ that satisfies:

$x_{i 2}^{*}=S o l\left[\begin{array}{lll}\operatorname{Max}_{x_{2} \geq 0} & u_{i 2}\left(x_{i 1}, x_{k l}, x_{i 2}, x_{k 2}^{*}\right) \\ \text { S.t.: } & G_{i 2}\left(x_{i 1}, x_{k 1}, x_{i 2}, x_{k 2}^{*}\right) \geq 0 & \left(\theta_{i}\right)\end{array}\right]$

$x_{k 2}^{*}=\operatorname{Sol}\left[\begin{array}{ccc}\operatorname{Max}_{x_{i 2} \geq 0} & u_{k 2}\left(x_{i l}, x_{k 1}, x_{i 2}^{*}, x_{k 2}\right) \\ \text { S.t.: } & G_{k 2}\left(x_{i 1}, x_{k l}, x_{i 2}^{*}, x_{k 2}\right) \geq 0 & \left(\theta_{k}\right)\end{array}\right]$

where $\theta_{i}$ and $\theta_{k}$ are the Lagrange multipliers associated with the respective constraints. Under the following assumptions, the Karush-Kuhn-Tucker (KKT) conditions for (2) can be used as an equivalent substitute for the period-two equilibrium problem.

Assumption 3: The KKT conditions for each of the respective agent's period-two optimization problems are necessary and sufficient.

Definition 4: The equivalent period-two equilibrium problem $E 2\left(x_{i 1}, x_{k 1}, x_{i 2}, x_{k 2}, \theta_{i}, \theta_{k}\right)$, abbreviated $E 2\left(x_{1}, x_{2}, \theta\right)$, consists of the KKT conditions for the agents' period-two optimization problems (2) for fixed $x_{k l}\left(x_{i l}\right)$ in agent i's (k's) problem:

$-\frac{\partial u_{i 2}}{\partial x_{i 2}}-\theta_{i}\left(\frac{\partial G_{i 2}}{\partial x_{i 2}}\right) \geq 0 \quad\left[\frac{\partial u_{i 2}}{\partial x_{i 2}}+\theta_{i}\left(\frac{\partial G_{i 2}}{\partial x_{i 2}}\right)\right] x_{i 2}=0 \quad \quad \mathrm{E} 2\left(\mathbf{x}_{1}, \mathbf{x}_{2}, \theta\right)$ $x_{i 2} \geq 0, \quad G_{i 2} \geq 0, \quad \theta_{i} \geq 0, \quad G_{i 2} \theta_{i}=0$ 
$-\frac{\partial u_{k 2}}{\partial x_{k 2}}-\theta_{k}\left(\frac{\partial G_{k 2}}{\partial x_{k 2}}\right) \geq 0 \quad\left[\frac{\partial u_{k 2}}{\partial x_{k 2}}+\theta_{k}\left(\frac{\partial G_{k 2}}{\partial x_{k 2}}\right)\right] x_{k 2}=0$

$x_{k 2} \geq 0, \quad G_{k 2} \geq 0, \quad \theta_{k} \geq 0, \quad G_{k 2} \theta_{k}=0$

Assumption 5: For every $\left(x_{i 1}, x_{k 1}\right)$ for which there exists a solution $\left(x_{i 2}, x_{k 2}, \theta_{i}, \theta_{k}\right)$ to $E 2\left(x_{1}, x_{2}\right.$, $\theta)$, that solution is unique.

Assumption 5 is sufficient to ensure that strategies are well defined solutions to $E 2\left(x_{1}, x_{2}, \theta\right)$ (that is, are functions) for fixed $\left(x_{i 1}, x_{k 1}\right)$, in that solutions in period-two variables are also consistent in both agents' period-two optimization problems.

Definition 6: Following (Ia), substitution of the equivalent period-two equilibrium conditions $E 2\left(x_{1}, x_{2}, \theta\right)$ into the period-one maximization problems of agents $i$ and $k$ yields the respective periodone maximization problems $P_{i}\left(x_{k \jmath}\right)$, in which $x_{k J}$ is a fixed parameter, and $P_{k}\left(x_{i j}\right)$, in which $x_{i j}$ is a fixed parameter:

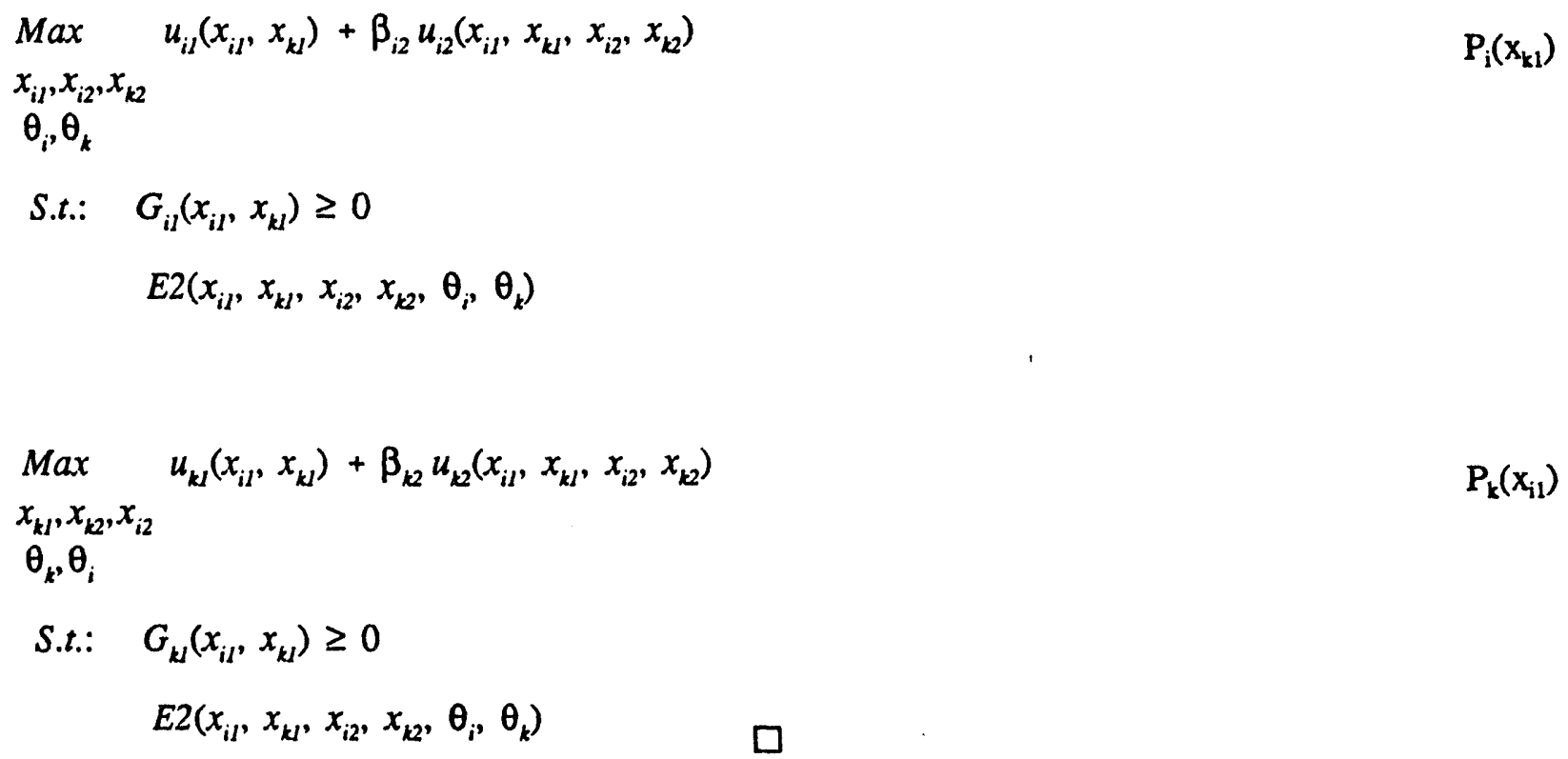


The two-agent, two-period SPE problem consists of simultaneously solving the two maximization problems $P_{i}\left(x_{k 1}\right)$ and $P_{k}\left(x_{i 1}\right)$. That is, an SPE solution $\left(x_{i 1}^{*}, x_{k 1}^{*}\right)$ satisfies:

$$
\begin{array}{ll}
x_{i l}^{*}=\operatorname{Sol}_{1} & P_{i l}\left(x_{k l}^{*}\right) \\
x_{k l}^{*}=\operatorname{Sol}_{1} & P_{k l}\left(x_{i j}^{*}\right)
\end{array}
$$

and under Assumption 5 it follows that $\mathrm{x}^{*}$ and $\mathrm{x}_{\mathrm{k} 2}^{*}$ satisfy (2) as required for an SPE solution.

An approach to simultaneously solving $P_{i}\left(x_{k 1}\right)$ and $P_{k}\left(x_{i 1}\right)$ is to combine the KKT conditions for these two problems and to solve the resulting nonlinear complementarity problem. The drawbacks of this approach are the difficulty of formulating the complementarity problems and the fact that the KKT conditions for $P_{i}\left(x_{k 1}\right)$ and $P_{k}\left(x_{i 1}\right)$ are necessary, but not sufficient, conditions for an optimal solution; that is, a solution to the complementarity problem is not necessarily an SPE solution. These approaches to solving static equilibrium formulations have been proposed by Harker $(1984,1986)$ and others. We are not aware of any research on solving dynamic equilibrium models formulated as mathematical programs other than that presented in this paper. The strategy described in the remainder of this paper simplifies the agent optimization problems by decomposing the feasible regions into convex subspaces, under appropriate assumptions.

\subsection{Characterization of Feasible Sets}

The strategy for solving the SPE problem is to first define a set $\Gamma$ that contains the feasible regions of both agents' period-one optimization problems. Each agent's optimization problem is defined over a different subset of $\Gamma$. Next, the set $\Gamma$ is divided into convex subsets $\Gamma_{f}$ and for each subset, a convex optimization problem is defined for each agent.

Definition 7: The set $\Gamma$ consists of points that satisfy $E 2\left(x_{1}, x_{2}, \theta\right)$ over the range of feasible $\left(x_{i l}, x_{k l}\right):$ 
$\Gamma=\left\{\left(x_{i j}, x_{k l}, x_{i 2}, x_{k 2}, \theta_{i}, \theta_{k}\right) \mid x_{i 1} \geq 0, x_{k l} \geq 0, G_{i l}\left(x_{1}\right) \geq 0, G_{k l}\left(x_{1}\right) \geq 0, E 2\left(x_{1}, x_{2}, \theta\right)\right\}$

\subsection{Facet Equilibrium}

Definition 8: $A$ facet index is an ordered $m$-tuple $(m>0)$ of binary variables $f=\left(f_{1} \ldots f_{m}\right)$ over the complementarity conditions in $E 2\left(x_{1}, x_{2}, \theta\right)$ where

$f_{j}=1$ if the $j^{\text {th }}$ complementarity variable $\geq 0$, and the $j^{\text {th }}$ complementarity constraint $=0$,

$f_{j}=2$ if the $j^{\text {th }}$ complementarity variable $=0$, and the $j^{\text {th }}$ complementarity constraint $\geq 0$.

The set of all facet indices is denoted by the set $F$.

A facet index can be used to denote a unique subset of the feasible regions in problems $P_{i}\left(x_{k 1}\right)$ and $P_{k}\left(x_{i 1}\right)$. The notion of a facet is defined for this purpose.

Definition 9: A facet $\Gamma_{f}, f \in F$, is the set of points which are feasible to the constraints of E2(x, $\left.x_{2}, \theta\right)$ indicated by facet index $f$ and the period-one constraints $x_{i l} \geq 0, x_{k l} \geq 0, G_{i l} \geq 0$, and $G_{k l} \geq 0$.

For example, facet $\Gamma_{1122}$ is the following set:

$$
\begin{gathered}
\Gamma_{1122}=\left\{\left(x_{i 1}, x_{k 1}, x_{i 2}, x_{k 2}, \theta_{i}, \theta_{k}\right) \mid x_{i 1} \geq 0, x_{k 1} \geq 0, G_{i l}\left(x_{1}\right) \geq 0, G_{k J}\left(x_{1}\right) \geq 0, E 2_{112}\left(x_{1}, x_{2}, \theta\right)\right\} \\
\text { where } E 2_{1122}\left(x_{1}, x_{2}, \theta\right) \equiv \\
\left\{x_{i 2} \geq 0, \quad-\frac{\partial u_{i 2}}{\partial x_{i 2}}-\theta_{i}\left(\frac{\partial G_{i 2}}{\partial x_{i 2}}\right)=0,\right. \\
x_{k 2} \geq 0, \quad-\frac{\partial u_{k 2}}{\partial x_{k 2}}-\theta_{k}\left(\frac{\partial G_{k 2}}{\partial x_{k 2}}\right)=0, \\
\theta_{i}=0, \quad G_{i 2} \geq 0, \\
\left.\theta_{k}=0, \quad G_{k 2} \geq 0\right\}
\end{gathered}
$$

In (3), the 1122 subscript on $\Gamma_{1122}$ indicates that the first member of the ccmplementary pairs in (3a) and (3b) have the inequality signs, and the second members in (3c) and (3d) have the inequality signs. 
The next two propositions follow directly from the definitions of $\Gamma, \Gamma_{n}$ and an SPE solution (see Macal and Hurter (1991) for complete proofs):

Proposition 10: $\Gamma=\cup_{f \in F} \Gamma_{f}$.

Proposition 11: Any subgame perfect equilibrium solution is a member of some facet $\Gamma_{f}, f \in F$. Facets are natural places to search for SPE solutions. Facet membership of an SPE outcome is not necessarily unique. Also, not every racet has a feasible point. For the sake of completeness, the notions of empty and nonempty facets are defined.

Definition 12: A point $\left(x_{1}, x_{2}, \theta\right)$ is feasible to facet $\Gamma_{f}, f \in F$, if $\left(x_{j}, x_{2}, \theta\right) \in \Gamma_{f}$. A facet $\Gamma_{f}$ is a nonempty facet if a point feasible to it exists. Otherwise $\Gamma_{f}$ is an empty facet.

The decomposition of the solution space into facets leads naturally to the formulation of equilibrium problems on facets, which are more easily solved than the original equilibrium problem. A facet optimization problem can be specified for each agent in period one:

Definition 13: For $f \in F$, the problems $P_{f(}\left(x_{k l}\right)$ and $P_{f k}\left(x_{i l}\right)$ are the period-one optimization problems on the facet $\Gamma_{f}$ :

$$
\begin{array}{ll}
\operatorname{Max}_{x_{i 1}, x_{i 2}, x_{k 2}} & u_{i l}\left(x_{i j}, x_{k j}\right)+\beta_{i 2} u_{i 2}\left(x_{i 1}, x_{k l}, x_{i 2}, x_{k 2}\right) \\
\theta_{i}, \theta_{k} & \\
\text { S.t.: } \quad x_{i l} \geq 0 & \\
& G_{i l}\left(x_{i j}, x_{k l}\right) \geq 0 \\
& E 2_{f}\left(x_{i l}, x_{k l}, x_{i 2}, x_{k 2}, \theta_{i}, \theta_{k}\right)
\end{array}
$$




$$
\begin{aligned}
& \operatorname{Max} \quad u_{k l}\left(x_{i 1}, x_{k j}\right)+\beta_{k 2} u_{k 2}\left(x_{i 1}, x_{k l}, x_{i 2}, x_{k 2}\right) \\
& x_{k 1}, x_{k 2}, x_{i 2} \\
& \theta_{k}, \theta_{i} \\
& \text { S.t.: } \quad x_{k\rfloor} \geq 0 \\
& G_{k J}\left(x_{i l}, x_{k J}\right) \geq 0 \\
& E 2_{f}\left(x_{i l}, x_{k l}, x_{i 2}, x_{k 2}, \theta_{i}, \theta_{k}\right)
\end{aligned}
$$

An equilibrium problem can be defined on each facet for each set of agent optimization problems.

Definition 14: For $f \in F$, the problem of simultaneously solving $P_{f i}\left(x_{k 1}\right)$ and $P_{f k}\left(x_{i 1}\right)$ on the facet $\Gamma_{f}$ is termed the facet equilibrium problem on facet $\Gamma_{f}$, or $f$. The solution of the facet equilibrium problem is a facet equilibrium solution (FES) on $\Gamma_{f}$.

For example, the simultaneous solution of $P_{1122, j}\left(x_{k 1}\right)$ and $P_{112, k}\left(x_{i 1}\right)$ for $\left(x_{1}^{*}, x_{2}^{*}\right)$ requires the following conditions to be satisfied:

$$
\begin{aligned}
& {\left[x_{i 1}^{*}, x_{i 2}^{*}, x_{k 2}^{*}\right]=\operatorname{Sol} P_{112, i}\left(x_{k l}^{*}\right)} \\
& {\left[x_{k 1}^{*}, x_{k 2}^{*}, x_{i 2}^{*}\right]=\operatorname{Sol} P_{112, k}\left(x_{i 1}^{*}\right)}
\end{aligned}
$$

To this point, we have redefined the SPE problem to be one of simultaneously solving a set of optimization problems. We next simplify the problem further by reducing the space of the decision variables to those only indexed on the first period.

\subsection{Common Solution Space}

The set $\Omega$ is the projection of the set $\Gamma$ onto the $x_{1}$ plane and consists of all the $x_{1}$ that are feasible to the period-one agent optimization problems $P_{i}\left(x_{k 1}\right)$ and $P_{k}\left(x_{i 1}\right)$ :

Definition 15: The period-one feasible projection set is: 
$\Omega=\left\{x_{1} \mid\right.$ there exists $\left(x_{2}, \theta\right)$ such that $\left.\left(x_{1}, x_{2}, \theta\right) \in \Gamma\right\}$

The period-one feasible projection set on facet $f \in F$ is defined analogously to $\Gamma_{f}$ :

$\Omega_{f}=\left\{x_{1} \mid\right.$ there exists $\left(x_{2}, \theta\right)$ such that $\left.\left(x_{1}, x_{2}, \theta\right) \in \Gamma_{f}\right\}$

Definition 16: The set $\Lambda$ consists of the original constraints of the SPE problem as follows:

$\Lambda=\left\{x_{1} \mid\right.$ there exists $x_{2}$ such that $\left.x_{1} \geq 0, x_{2} \geq 0, G_{1}\left(x_{1}\right) \geq 0, G_{2}\left(x_{1}, x_{2}\right) \geq 0\right\}$

Because $\Lambda$ is defined in terms of the original constraints of the SPE problem, properties of $\Lambda$ are easier to verify than properties of the sets $\Omega_{\mathrm{f}}$ and $\Omega$. Assumption 5 can be restated in light of the definition of $\Lambda$ :

Assumption 17: For every $x_{1} \in \Lambda$ there exists a unique solution $\left[x_{2}\left(x_{1}\right), \theta\left(x_{1}\right)\right]$ to the equivalent period-two equilibrium problem E2( $\left.x_{1}, x_{2}, \theta\right)$.

\subsection{Numerical Example}

Figure 2 illustrates the set $\Omega_{1122}$ for a numerical example with the following parameters:

$G_{i d}\left(x_{1}\right): \varnothing$

$G_{k j}\left(x_{1}\right): \varnothing$

$G_{i 2}\left(x_{1}, x_{2}\right): B_{i}-x_{i 1}-x_{i 2} \geq 0, \quad B_{i}=97$

$G_{k 2}\left(x_{1}, x_{2}\right): B_{k}-x_{k l}-x_{k 2} \geq 0, B_{k}=52$

$$
\begin{array}{rlrl}
E 2\left(x_{1}, x_{2}, \theta\right): & u=14 x_{i 2}+2 x_{k 2}+10 x_{i 1}-1000 \geq 0, & x_{i 2} \geq 0, & u x_{i 2}=0 \\
v & =5 x_{k 2}+2 x_{i 2}+x_{k l}-100 \geq 0, & x_{k 2} \geq 0, & v x_{k 2}=0 \\
w=97-x_{i 1}-x_{i 2} \geq 0, & \theta_{i} \geq 0, & w \theta_{i}=0 \\
z=52-x_{k 1}-x_{k 2} \geq 0, & \theta_{k} \geq 0, & z \theta_{k}=0
\end{array}
$$


This example consists of an oligopolistic market model with linear demand, quadratic costs, and resource constraints that limit the total amount of resource producible by each agent over the two periods. To construct $\Omega_{1122}$, derive $E 2_{1122}\left(x_{1}, x_{2}, \theta\right)$ from $E 2\left(x_{1}, x_{2}, \theta\right)$ :

$$
\begin{array}{lll}
14 x_{i 2}+2 x_{k 2}+10 x_{i 1}-1000=0, & x_{i 2} \geq 0 & \mathrm{E} 2_{1122}\left(\mathrm{x}_{1}, \mathrm{x}_{2}, \theta\right) \\
5 x_{k 2}+2 x_{i 2}+x_{k 1}-100=0, & x_{k 2} \geq 0 & \\
97-x_{i 1}-x_{i 2} \geq 0, & \theta_{i}=0 \\
52-x_{k 1}-x_{k 2} \geq 0, & \theta_{k}=0 &
\end{array}
$$

Solving $\mathrm{E} 2_{1122}\left(\mathrm{x}_{1}, \mathrm{x}_{2}, \theta\right)$ for $\mathrm{x}_{2}$ in terms of $\mathrm{x}_{1}$ gives the strategy mappings $\mathrm{X}_{\mathrm{i2}}\left(\mathrm{x}_{\mathrm{i} 1}, \mathrm{x}_{\mathrm{k} 1}\right)$ and $\mathrm{X}_{\mathrm{k} 2}\left(\mathrm{x}_{\mathrm{i1}}, \mathrm{x}_{\mathrm{k} 1}\right)$ :

$$
\begin{aligned}
& X_{i 2}\left(x_{i l}, x_{k l}\right)=-0.75758 x_{i 1}+0.03030 x_{k l}+72.72727 \geq 0 \\
& X_{k 2}\left(x_{i 1}, x_{k l}\right)=0.30303 x_{i l}-0.21212 x_{k l}-9.09091 \geq 0
\end{aligned}
$$

where the inequalities follow from the non-negativity constraints on $x_{i 2}$ and $x_{k 2}$, respectively.

Substituting $X_{i 2}\left(x_{i 1}, x_{k 1}\right)$ and $X_{k 2}\left(x_{i 1}, x_{k 1}\right)$ for $x_{i 2}$ and $x_{k 2}$, respectively, into the $G_{2}$ constraints together with the non-negativity constraints on $\mathrm{x}_{i 2}$ and $\mathrm{x}_{k 2}$ yields the set $\Omega_{1122}$ :

$$
\begin{gathered}
\Omega_{1122}=\left\{\left(x_{i l}, x_{k l}\right) \geq 01 \quad 2400-25 x_{i 1}+x_{k l} \geq 0,-300+10 x_{i 1}-7 x_{k l} \geq 0,\right. \\
\left.801-8 x_{i 1}-x_{k J} \geq 0,1008-5 x_{i J}-13 x_{k l} \geq 0\right\}
\end{gathered}
$$

For all $\mathrm{f} \in \mathrm{F}=\left\{\left(\begin{array}{llll}1 & 1 & 1 & 1\end{array}\right),\left(\begin{array}{llll}1 & 1 & 1 & 2\end{array}\right), \ldots,\left(\begin{array}{lll}2 & 2 & 2\end{array}\right)\right\}$, the sets $\Omega_{f}$ are constructed similarly to $\Omega_{1122}$. Figure 3 displays the sets $\Omega_{f}, \Omega$, and $\Lambda=\left\{\left(x_{i 1}, x_{k 1}\right) \mid 0 \leq x_{i 1} \leq 97,0 \leq x_{k 1} \leq 52\right\}$. Note, that $\Omega_{f}, f \in$ F, tiles the set $\Omega$. Some $\Omega_{\mathrm{f}}$ are empty sets as no point satisfies all of the equations and inequalities defining the facet $\Gamma_{\mathrm{f}}$. Also, some $\Omega_{\mathrm{f}}$ are not of full dimension two, where full dimension is the dimension of the set $\Omega$, the length of the $x_{1}$ vector, or the number of agents. These sets include $\Omega_{1221}$ of dimension one, a line in $\mathbf{R}^{2}$, and $\Omega_{2211}$ of dimension zero, a point in $\mathbf{R}^{2}$. 
We have completed the decomposition of the space into convex subspaces parameterized on $x_{1}$. Any equilibrium solution on a facet $f$ can be defined as any $\left(x_{i 1}, x_{k 1}\right)$ pair that simultaneously solves $P_{6}\left(x_{k 1}\right)$ and $P_{k k}\left(x_{i 1}\right)$. The transformation $P_{f}\left(x_{1}\right) \equiv\left[P_{f 1}\left(x_{k 1}\right), P_{k k}\left(x_{i 1}\right)\right]$ takes any point $x_{1} \in \Omega_{q}$ and maps it to another point in the same convex space $\Omega_{f}$; that is $P_{f}: \Omega_{f} \rightarrow \Omega_{f}, f \in F$. The properties of a mapping based on $P_{f}$ and the existence of facet equilibrium solutions are addressed in Section 2 .

\section{EXISTENCE OF FACET EQUILIBRIUM SOLUTIONS}

We will show that every nonempty facet contains a facet equilibrium solution, as stated in the following theorem.

Theorem 18: Let $\Gamma_{f}$ be a nonempty facet, $f \in F$, and the functions defining the facet be linear (as in the case of $U$ quadratic) and the problem constraints $G$ linear. Let Assumptions 3 and 17 hold. Then there exists a facet equilibrium solution on facet $\Gamma_{f}$.

Theorem 18 will be proved in the following steps:

1. Show that $\Omega_{\mathrm{f}}$ is a convex and compact set under appropriate assumptions on $G_{i}$ and $G_{k}$.

2. Define a function $T_{f}$, based on $P_{f}$, over the set $\Omega_{f}$ for any nonempty facet.

3. Show that $T_{f}$ is a continuous mapping on $\Omega_{\mathrm{f}}$.

4. Show that a fixed point of $T_{f}$ exists and is a facet equilibrium solution.

It follows from Theorem 18 that any SPE solution is a fixed point of the mapping $T_{f}$ for some $f \in F$. Therefore, it is natural to compute fixed points of $T_{f}$ and test whether they are SPE solutions. This procedure will be described in Section 3.

\subsection{Properties of the Feasible Space}

Lemma 19: If Assumption 17 holds, then $\Lambda=\Omega$.

Proof that $\Omega \subseteq \Lambda$ : Let $x_{1} \in \Omega$. Then $x_{1} \in \Omega_{\mathrm{f}}$ for some $f \in F$. By the definition of $\Omega_{\mathrm{f}}$, there exists $\left(x_{2}, \theta\right)$ with $\left(x_{1}, x_{2}, \theta\right) \in \Gamma_{f}$. Hence, $\left(x_{1}, x_{2}, \theta\right)$ satisfies the constraints $\left\{x_{1} \geq 0, x_{2} \geq 0, G_{i} \geq 0\right.$, 
$\left.G_{k} \geq 0\right\}$ and $E 2\left(x_{1}, x_{2}, \theta\right)$. Thus, given $x_{1}$, there exists $x_{2}$ such that $\left(x_{1}, x_{2}\right)$ satisfies the set of constraints $\left\{x_{1} \geq 0, x_{2} \geq 0, G_{i} \geq 0, G_{k} \geq 0\right\}$. This implies that $x_{1} \in \Lambda$.

Proof that $\Lambda \subseteq \Omega$ : Let $x_{1} \in \Lambda$. By Assumption 17 there exists a solution to $E 2\left(x_{1}, x_{2}, \theta\right)$. Denote this solution by $\left[x_{2}\left(x_{1}\right), \theta\left(x_{1}\right)\right]$. Then $\left[x_{1}, x_{2}\left(x_{1}\right), \theta\left(x_{1}\right)\right] \in \Gamma_{\ell}$ for some $f \in F$ because $\cup_{f \in F} \Gamma_{\ell} \subseteq$ $\Gamma$. Hence, $x_{1} \in \Omega_{\mathrm{f}} \subseteq \Omega$.

We next make an assumption regarding the compactness of the constraint regions of the agent optimization problems of the SPE problem.

Assumption 20: $\Lambda$ is a compact (closed and bounded) set in $\boldsymbol{R}^{n}$.

It follows from Assumptions 17 and 20 that $\Omega$ is a compact set. The next proposition follows from the assumption of the compactness of $\Lambda$ and the results that $\Lambda=\Omega, \Gamma_{f}$ is a (closed, compact) polyhedron in $\mathbf{R}^{\mathrm{n}}$, and $\Omega=\cup_{\mathrm{f} \in \mathrm{F}} \Omega_{\mathrm{f}}$. A complete proof is given in Macal (1989).

Proposition 21: If $\Lambda$ is a compact (closed and bounded) set in $\boldsymbol{R}^{n}$, then $\Omega_{f}$ is compact and convex for $f \in F$.

The next section defines the mapping $T_{\mathrm{f}}$ over the search space $\Omega_{\mathfrak{q}}$.

\subsection{The Mapping $T_{f}$}

Definition 22: For any nonempty facet $\Gamma_{f}$, define a vector mapping $T_{f}$ on $\Omega_{f}$ as $T_{f}\left(x_{i l}, x_{k l}\right)=\left[T_{f i}\right.$ $\left.\left(x_{i l}, x_{k l}\right), T_{f k}\left(x_{i l}, x_{k l}\right)\right]$ where for any fixed $\lambda \in(0,1)$

$$
\begin{aligned}
& T_{f i}\left(x_{i l}, x_{k l}\right) \equiv \lambda x_{i l}+(1-\lambda) x_{i l}\left(x_{k l}\right), \text { and } \\
& T_{f k}\left(x_{i l}, x_{k l}\right) \equiv \lambda x_{k l}+(1-\lambda) x_{k l}\left(x_{i l}\right)
\end{aligned}
$$

with $x_{i l}\left(x_{k l}\right)$ and $x_{k l}\left(x_{i l}\right)$ defined as:

$$
\begin{aligned}
& x_{i l}\left(x_{k l}\right) \equiv \operatorname{Sol}_{1} P_{f i}\left(x_{k l}\right), \text { and } \\
& x_{k l}\left(x_{i l}\right) \equiv \operatorname{Sol}_{l} P_{f k}\left(x_{i 1}\right) . \square
\end{aligned}
$$


The function $\left[x_{i 1}\left(x_{k 1}\right), x_{k 1}\left(x_{i 1}\right)\right]$ is the response function that given an input $\left(x_{i 1}, x_{k 1}\right)$ produces a result for the period-one agent optimization problems.

Assumption 23: Let $\Gamma_{f}$ be a nonempty facet. For any $\left(x_{i j}, x_{k l}\right) \in \Omega_{f}$ there exists a unique solution for $x_{i 1}$ to $P_{f l}\left(x_{k 1}\right)$ and there exists a unique solution for $x_{k l}$ to $P_{f k}\left(x_{i l}\right)$. That is $x_{i l}\left(x_{k l}\right)=\operatorname{Sol}_{1} P_{f i}\left(x_{k 1}\right)$ and $x_{k 1}\left(x_{i l}\right)=\operatorname{Sol}_{1} P_{f_{k}}\left(x_{i l}\right)$ are unique given $\left(x_{i l}, x_{k l}\right)$.

The numerical example of the previous section satisfies Assumption 23. Next it will be shown that $T_{f}$ is a continuous mapping of the set $\Omega_{\mathrm{f}}$ into itself.

Theorem 24: Let $\Gamma_{f}$ be a nonempty facet. Then $T_{f}$ maps $\Omega_{f}$ into itself. That is, $T_{f}: \Omega_{f} \rightarrow \Omega_{f}$.

Proof: Let $x_{1} \in \Omega_{q}$. The theorem requires that $T_{f}\left(x_{1}\right) \in \Omega_{f}$. Let

$$
\begin{aligned}
& {\left[x_{i 1}\left(x_{k 1}\right), x_{i 2}\left(x_{k 1}\right), x_{k 2}\left(x_{k 1}\right), \theta_{i}\left(x_{k 1}\right), \theta_{k}\left(x_{k 1}\right)\right] \equiv \text { Sol } P_{f i}\left(x_{k 1}\right), \text { and }} \\
& {\left[x_{k 1}\left(x_{i 1}\right), x_{k 2}\left(x_{i 1}\right), x_{i 2}\left(x_{i 1}\right), \theta_{i}\left(x_{i 1}\right), \theta_{k}\left(x_{i 1}\right)\right] \equiv \text { Sol } P_{k k}\left(x_{i 1}\right) .}
\end{aligned}
$$

To prove that $T_{f}\left(x_{i 1}, x_{k 1}\right) \in \Omega_{f}$, it is sufficient to show that

$$
\left[\lambda \mathrm{x}_{\mathrm{i} 1}+(1-\lambda) \mathrm{x}_{\mathrm{i} 1}\left(\mathrm{x}_{\mathrm{k} 1}\right), \lambda \mathrm{x}_{\mathrm{k} 1}+(1-\lambda) \mathrm{x}_{\mathrm{k} 1}\left(\mathrm{x}_{\mathrm{i} 1}\right)\right] \in \Omega_{\mathrm{f}} .
$$

Because Sol $P_{f 1}\left(x_{k 1}\right)$ is optimal to problem $P_{f}\left(x_{k 1}\right)$, it is also feasible to the constraints of $P_{6}\left(x_{k 1}\right)$. Hence

$$
\left[x_{i 1}\left(x_{k 1}\right), x_{k 1}, x_{i 2}\left(x_{k 1}\right), x_{k 2}\left(x_{k 1}\right), \theta_{i}\left(x_{k 1}\right), \theta_{k}\left(x_{k 1}\right)\right] \in \Gamma_{f} .
$$

Similarly for agent k's problem

$$
\left[x_{i 1}, x_{k 1}\left(x_{i 1}\right), x_{i 2}\left(x_{i 1}\right), x_{k 2}\left(x_{i 1}\right), \theta_{i}\left(x_{i 1}\right), \theta_{k}\left(x_{i 1}\right)\right] \in \Gamma_{f} .
$$

Note that $\left[x_{i 1}\left(x_{k 1}\right), x_{k 1}\right] \in \Omega_{\mathrm{f}}$ and $\left[x_{i 1}, x_{k 1}\left(x_{i 1}\right)\right] \in \Omega_{\mathrm{f}}$. Since $\Omega_{\mathrm{f}}$ is convex by Proposition 21 , it follows that for any $\lambda \in(0,1)$

$$
\left[\lambda x_{i 1}+(1-\lambda) x_{i 1}\left(x_{k 1}\right), \lambda x_{k 1}+(1-\lambda) x_{k 1}\left(x_{i 1}\right)\right] \in \Omega_{\mathrm{f}} .
$$

\subsection{Continuity of $T_{\text {, }}$}

The next step in the proof of Theorem 18 is to show that $T_{f}$ is a continuous mapping of the parameters $\left(x_{i 1}, x_{k 1}\right)$. A theorem by Fiacco is instrumental in this development. 
Theorem (Fiacco, 1983): Consider the following mathematical program:

$\operatorname{Min}_{x} q(x, \varepsilon)$

S.t.: $\quad g(x, \varepsilon) \geq 0$,

$h(x, \varepsilon)=0$,

where the problem functions $q, g$, and $h$ are continuously differentiable in $(x, \varepsilon)$, and

$$
R(\varepsilon) \equiv\left\{x \in \boldsymbol{R}^{n} / g(x, \varepsilon) \geq 0, h(x, \varepsilon)=0\right\}
$$

is defined to be the feasible region of $P 3(\varepsilon)$,

$$
S(\varepsilon) \equiv\left\{x \in R(\varepsilon) / q(x)=q^{*}(\varepsilon)\right\}
$$

is defined to be the set of optimal solutions to problem $P 3(\varepsilon)$ above,

$$
q^{*}(\varepsilon)=\inf _{x}\{q(x) / x \in R(\varepsilon)\}
$$

is the optimal value function as $\varepsilon$ varies, and the parameter $\varepsilon$ is a vector in $\boldsymbol{R}^{\boldsymbol{n}}$. Then, if $R(0) \neq \varnothing$, with $R$ uniformly compact near $\varepsilon=0$, and if the Mangasarian-Fromovitz constraint qualification (Mangasarian and Fromovitz, 1967) holds for some $\dot{x}^{*} \in S(0), q^{*}$ is continuous at $\varepsilon=0$.

In the facet equilibrium problem, $U$ quadratic and $G$ linear imply that the functions $h_{j}$ and $g_{i}$ above are affine. In tum $g_{i}$ affine implies that $g_{i}$ is concave and is therefore pseudo-concave (Fiacco, 1983). Based on these observations and following Fiacco, the facts that $g_{i}$ is pseudo-concave and $h_{j}$ is affine imply that the Mangasarian-Fromovitz constraint qualification is equivalent to the Slater condition, a general form of which is as follows:

Constraint Qualification (Slater, 1950): The Slater constraint qualification is satisfied at $x^{*} \in R$ $\equiv\left\{x \in \boldsymbol{R}^{n} / g(x) \geq 0, h(x)=0\right\}$ if $h_{j}$ is affine for each $j, g_{i}$ is pseudo-concave for each $i$ and there exists a point $y \in R$ with $g_{i}(y)>0$ for each $i$ in $B\left(x^{*}\right) \equiv\left\{i / g_{i}\left(x^{*}\right)=0\right\}$.

Theorem 25: Assume that Assumptions 5 and 17 and the Slater constraint qualification hold for each agent's period-one optimization problem on the nonempty facet $\Gamma_{f}$. Denote $V_{f(}\left(x_{k l}\right)$ as the optimal 
profit level for agent $i$ obtainable on a nonempty facet $f$ as a function of the parameter $x_{k j}$. That is

$$
V_{f 1}\left(x_{k 1}\right)=U_{i 1}\left(x_{i 1}\left(x_{k l}\right), x_{k 1}, x_{i 2}\left(x_{k 1}\right), x_{k 2}\left(x_{k 1}\right)\right)
$$

where $\left[x_{i 1}\left(x_{k 1}\right), x_{i 2}\left(x_{k 1}\right), x_{k 2}\left(x_{k 1}\right)\right]=$ Sol $P_{f 1}\left(x_{k 1}\right)$. Then by Fiacco's theorem $V_{f 1}\left(x_{k 1}\right)$ is a continuous mapping of $x_{k l}$.

Proof: Relate the variables of the SPE problem to the variables of Fiacco's Theorem as follows. Take $P_{f 1}\left(x_{k 1}\right)$ for $P 3(\varepsilon)$ with

$$
\begin{aligned}
& x=\left(x_{i 1}, x_{i 2}, x_{k 2}, \theta_{i}, \theta_{k}\right), \\
& \varepsilon=x_{k 1}, \\
& q(x, \varepsilon)=U_{i}\left(x_{i 1}, x_{i 2}, x_{k 2}, x_{k 1}\right), \\
& h(x, \varepsilon)=\text { equality constraints defining facet } \Gamma_{\mathfrak{f}} \text { for } x_{k 1} \text { fixed, } \\
& g(x, \varepsilon)=\text { inequality constraints defining facet } \Gamma_{t} \text { for } x_{k 1} \text { fixed, } \\
& R(\varepsilon)=\text { feasible region of agent i's period-one optimization problem on facet } \Gamma_{t} \text { for } x_{k 1} \text { fixed, } \\
& S(\varepsilon)=\text { set of optimal solutions to problem } P_{f 1}\left(x_{k 1}\right) .
\end{aligned}
$$

Since $\Gamma_{\mathrm{f}}$ is nonempty by assumption, the feasible region $R(\varepsilon) \neq \varnothing . U_{i}$ and the constraint functions are continuously differentiable for any $f \in F$. All inequality constraints of the original SPE problem are linear, thus $g_{i}$ is pseudo-concave. Under the assumption that $U_{i}$ is quadratic, $h_{j}$ is affine. It can be shown that $\mathbf{R}\left(\mathbf{x}_{\mathbf{k} 1}\right)$ is uniformly compact (Macal, 1989). Therefore by Fiacco's theorem, $V_{\mathrm{ak}}$ is a continuous mapping of $x_{k 1}$. The continuity of $V_{f i}$ on $x_{i 1}$ follows by a similar argument.

Theorem 25 used Fiacco's theorem to show that the optimal objective function value for agent $\mathrm{i}$ (agent $k$ ) on any nonempty facet is a continuous function of the parameter $x_{k 1}$ (parameter $x_{i 1}$ ). Theorem 26 will use this result to show that the optimal solution to agent i's (agent k's) optimization problem is a continuous function of $x_{k 1}$ (of $x_{i 1}$ ) on any nonempty facet. This result will be used to show that $T_{f}$ is a continuous mapping. 
Theorem 26: Assume that Assumptions 5 and 17 hold. Also assume that the Slater constraint qualification holds for each agent's period-one optimization problem on the nonempty facet $\Gamma_{f}$. Then $x_{i l}\left(x_{k 1}\right)$ is a continuous mapping of $x_{k l}$ where $x_{i j}\left(x_{k j}\right)=\operatorname{Sol}_{1} P_{f 1}\left(x_{k l}\right)$.

Proof: The proof of continuity of the optimal solutions follows Murphy et al. (1982, Lemma 2). For simplicity of exposition denote the following:

$$
\begin{aligned}
& x=\left(x_{i 1}, x_{i 2}, x_{k 2}\right), \\
& \chi\left(x_{k 1}\right)=\left[x_{i 1}\left(x_{k 1}\right), x_{i 2}\left(x_{k 1}\right), x_{k 2}\left(x_{k 1}\right)\right], \text { and } \\
& \theta\left(x_{k 1}\right)=\left[\theta_{i}\left(x_{k 1}\right), \theta_{k}\left(x_{k 1}\right)\right] .
\end{aligned}
$$

Let $\left[\chi\left(x_{k 1}\right), \theta\left(x_{k 1}\right)\right]$ denote the optimal solution to $P_{f 1}\left(x_{k 1}\right)$ given $x_{k 1}$, and let $V_{i}\left(x_{k 1}\right)$ denote the optimal objective function value as $\mathbf{x}_{\mathbf{k} 1}$ varies:

$$
V_{i}\left(x_{k 1}\right)=U_{i}\left(\chi\left(x_{k 1}\right), x_{k 1}\right) \text {. }
$$

Theorem 25 showed that $V_{i}\left(x_{k 1}\right)$ is a continuous function of $x_{k 1}$. Thus, given any feasible convergent sequence on the facet $\Gamma_{f},\left[x_{i 1}\left(x_{k 1}^{n}\right), x_{k 1}^{n}\right] \in \Omega_{f}$ with $\left\{x_{k 1}^{n}\right\}_{n} \rightarrow x_{k 1}$, it follows that

$$
\left\{V_{i}\left(x_{k 1}^{n}\right)\right\}_{n} \equiv\left\{U_{i}\left(\chi\left(x_{k 1}^{n}\right), x_{k 1}^{n}\right)\right\}_{n} \rightarrow V_{i}\left(x_{k 1}\right) \equiv U_{i}\left(\chi\left(x_{k 1}\right), x_{k, 1}\right)
$$

The sequence $\left\{\chi\left(x_{k 1}^{n}\right)\right\}_{n}$ is bounded by the compactness of $\Omega_{f}$ (Proposition 21) and Assumption 17 regarding the existence of a solution to $E 2\left(x_{1}\right)$ for every $x_{1} \in \Lambda$. The sequence therefore has a convergent subsequence. If $\mathrm{n}$ indexes such a sequence, there exist limits as follows:

$$
\left[x_{i 1}\left(x_{k 1}^{n}\right), x_{i 2}\left(x_{k 1}^{n}\right), x_{k 2}\left(x_{k 1}^{n}\right)\right] \rightarrow\left(y_{i 1}, y_{i 2}, y_{k 2}\right) \text {. }
$$

That is, $\lim _{n} \chi\left(x_{k !}^{n}\right) \equiv\left(y_{i 1}, y_{i 2}, y_{12}\right)$. Since

$$
\left[x_{i 1}\left(x_{k 1}^{n}\right), x_{k 1}^{n}, x_{i 2}\left(x_{k 1}^{n}\right), x_{k 2}\left(x_{k 1}^{n}\right), \theta_{i}\left(x_{k 1}^{n}\right), \theta_{k}\left(x_{k 1}^{n}\right)\right] \in \Gamma_{t} \text { for each } n \text { it follows that }\left(y_{i 1}, y_{k 1}, y_{i 2}, y_{k 2}\right. \text {, }
$$
$\theta_{i}\left(x_{k 1}\right), \theta_{k}\left(x_{k 1}\right) \in \Gamma_{t}$ from the definition of $\Gamma_{t}$ and the fact that for any continuous functions $f$ and $g$, $\mathrm{f}\left(\varepsilon_{\mathrm{n}}\right)=0, \mathrm{~g}\left(\varepsilon_{n}\right) \geq 0$, and $\varepsilon_{\mathrm{n}} \rightarrow \varepsilon$ with $\mathrm{n} \rightarrow \infty$ implies that $\mathrm{f}(\varepsilon)=0$ and $\mathrm{g}(\varepsilon) \geq 0$. That is, the limit point $\left(y_{i 1}, y_{k 1}, y_{i 2}, y_{k 2}, \theta_{i}\left(x_{k 1}\right), \theta_{k}\left(x_{k 1}\right)\right)$ is feasible to $\left.P_{f 1}\left(y_{k 1}\right)\right)$. Suppose that the solutions to problem 
$P_{\mathfrak{n}}\left(\mathbf{x}_{\mathbf{k} 1}\right)$ are not continuous:

$\left(y_{i 1}, y_{i 2}, y_{k 2}\right) \neq\left[x_{i 1}\left(x_{k 1}\right), x_{i 2}\left(x_{k 1}\right), x_{k 2}\left(x_{k 1}\right)\right]$

From (4) above

$$
\begin{aligned}
& U_{i}\left(\chi\left(x_{k 1}\right), x_{k 1}\right)=\lim _{n} U_{i}\left(\chi\left(x_{k 1}^{n}\right), x_{k 1}^{n}\right) \\
& =U_{i}\left(\lim _{n} \chi\left(x_{k 1}^{n}\right), \lim _{n} x_{k 1}^{n}\right) \\
& =U_{i}\left(y_{i 1}, y_{i 2}, y_{k 2}, y_{k 1}\right)
\end{aligned}
$$

where (5) follows from the continuity of $U_{i}$ in all variables and (6) follows from (4). Equation 6 contradicts the uniqueness of $\chi\left(x_{k 1}\right)=\left[x_{i 1}\left(x_{k 1}\right), x_{i 2}\left(x_{k 1}\right), x_{k 2}\left(x_{k 1}\right)\right]$, Assumption 17, for the given $x_{k 1}$. Hence $x_{i 1}\left(x_{k 1}\right)$ is continuous for all $x_{k 1}$ such that $\left[x_{i 1}\left(x_{k 1}\right), x_{k 1}\right] \in \Omega_{1}$. The continuity of $x_{k 1}\left(x_{i 1}\right)$ follows by a similar argument. The continuity of $T_{f}$ follows since $T_{f}$ is a continuous function of $x_{i 1}\left(x_{k 1}\right)$ and $\mathrm{x}_{\mathrm{k} 1}\left(\mathrm{x}_{\mathrm{i} 1}\right)$

Next it will be shown that there exists a fixed point of $T_{f}$ in $\Omega_{f}$ and that this fixed point is a facet equilibrium solution.

\subsection{Existence of a Facet Equilibrium Solution}

Theorem 27: For any nonempty facet $\Gamma_{f}, f \in F$, there exists a fixed point of $T_{f}$ in $\Omega_{f}$.

Proof: By Brouwer's Theorem (Brouwer, 1910), any continuous mapping $H$ that maps a nonempty, compact, and convex set $D \subseteq \mathbf{R}^{\mathrm{n}}$ into itself, $\mathrm{H}: \mathrm{D} \rightarrow \mathrm{D}$, has a fixed point $\mathrm{x} \in \mathrm{D}$ such that $x=H(x)$. Applying Brouwer's Theorem with $D=\Omega_{f}, \Gamma_{f}$ nonempty, and $H=T_{t}$, it follows by Proposition 21 that $\Omega_{f}$ is compact and convex. By Theorem 26, $T_{f}$ is continuous, and by Theorem 24 , $T_{f}$ maps $\Omega_{q}$ into itself. Hence, there exists some $\left(x_{i 1}, x_{k 1}\right) \in \Omega_{q}$ such that $\left(x_{i 1}, x_{k 1}\right)=T_{f}\left(x_{i 1}, x_{k 1}\right)$.

Theorem 28: Any fixed point of $T_{f}$ in $\Omega_{f}$ for $f \in F$ is a facet equilibrium solution.

Proof: Let $\left(x_{i 1}, x_{k 1}\right)$ be a fixed point of $T_{f}$ in $\Omega_{q}$ such that $x_{i 1}=T_{k i}\left(x_{i 1}, x_{k 1}\right)$ and $x_{k 1}=T_{k k}\left(x_{i 1}, x_{k 1}\right)$. By the definition of $T_{t}$ it follows that 


$$
\begin{aligned}
& (1-\lambda) x_{i 1}=(1-\lambda) x_{i 1}\left(x_{k 1}\right), \text { and } \\
& (1-\lambda) x_{k 1}=(1-\lambda) x_{k 1}\left(x_{i 1}\right)
\end{aligned}
$$

which implies for $\lambda \in(0,1)$ that

$$
\begin{aligned}
& x_{i 1}=x_{i 1}\left(x_{k 1}\right), \text { and } \\
& x_{k 1}=x_{k 1}\left(x_{i 1}\right) \cdot \square
\end{aligned}
$$

This completes the proof of Theorem 18 conceming the existence of an FES on every nonempty facet.

\subsection{Implications for Subgame Perfect Equilibrium Solutions}

We will show that any SPE solution is in the set of FESs.

Theorem 29: Define the following sets: $F a c E\left(\Omega_{f}\right)=$ set of $F E S$ s in the set $\Omega_{f}$, and $S P E(\Omega)=$ set of $S P E$ solutions in the set $\Omega$. Then, $S P E(\Omega) \subseteq U_{f \in F} F a c E\left(\Omega_{f}\right)$.

Proof: If $x_{1} \in F a c E\left(\Omega_{f}\right)$, then $x_{1}$ is a globally maximizing solution over $\Omega_{f}$ for each agent. That is, $x_{i 1}$ solves $P_{f i}\left(x_{k 1}\right)$ given $x_{k 1}$, and $x_{k 1}$ solves $P_{k k}\left(x_{i 1}\right)$ given $x_{i 1}$. For $x_{1}$ to be an SPE solution it is necessary that $x_{1}$ is a globally maximizing solution over the entire set $\Omega$ for each agent. Not all $x_{1}$ that are globally maximizing over $\Omega_{\mathrm{f}}$ are also globally maximizing over the set $\Omega$ because $\Omega=\cup_{\mathrm{NF}} \Omega_{\mathrm{f}}$; a point $x_{1} \in \operatorname{FacE}\left(\Omega_{\mathrm{f}}\right)$ could be globally maximizing, locally maximizing, a stationary point, or none of these with respect to the set $\operatorname{SPE}(\Omega)$. On the other hand, any SPE solution is maximizing for each agent over the set $\Omega$ and therefore is maximizing for each agent over $\Omega_{\mathrm{f}}$ for some f, again because $\Omega=\cup_{f \in F} \Omega_{\mathrm{f}}$.

Theorem 29 has implications for a strategy to compute SPE solutions: compute FESs and test whether each solution is also an SPE solution. The next section describes a computational procedure for searching for SPE solutions. 


\section{SOLUTION ALGORITHM}

The search for SPE solutions is conducted over the finite set of facets.

\subsection{Steps of the Algorithm}

The steps of the algorithm are illustrated in Figure 4:

Step 1: Find initial facet. Find a feasible starting facet projection set $\Omega_{\mathrm{r}}$ for some $f \in F$.

Step 2: Solve facet equilibrium problem. Solve the facet equilibrium problem on $\Omega_{\ell}$ for an FES. This FES is a candidate for an SPE solution. There are three mutually exclusive and exhaustive cases for an FES. Typical examples of the cases are illustrated in Figure 5 and are described as follows.

Case 1: The FES is strictly interior to $\Omega_{\mathrm{f}}$. In this case, it follows that the FES is strictly interior to $\Omega$. By the definition of a facet, a point that is strictly interior to a facet projection $\Omega_{\mathrm{f}}$ is not contained in any facet projection other than $\Omega_{\mathfrak{f}}$. In this case the FES is a local optimum for each agent with respect to the set $\Omega$. However, an SPE solution is globally optimal for each agent on the set $\Omega$. In this case, proceed to Step 3 and check whether the FES that is locally optimai on $\Omega$ is also globally optimal for each agent.

Case 2: The FES is on the boundary of $\Omega_{f}$ and is not contained in any facet projection other than $\Omega_{\mathrm{f}}$. In this case, the FES is also contained in the boundary of $\Omega$ and is a local optimum for each agent with respect to the set $\Omega$. An SPE solution is globally optimal for each agent on the set $\Omega$. Therefore, proceed to Step 3 and check whether the FES that is locally optimal on $\Omega$ is also globally optimal for each agent.

Case 3: The FES is on the boundary of $\Omega_{\mathrm{f}}$ and is contained in some facet projection other than $\Omega_{f}$, say the facet projection $\Omega_{g}$, for $g \neq f$. In this case, the FES for $\Omega_{f}$ is also contained in the boundary of the adjacent facet projection set $\Omega_{\mathrm{g}}$. Although the FES is a local optimum for each agent 
with respect to the set $\Omega_{\mathrm{f}}$, it is not necessarily a local optimum for each agent with respect to the set $\Omega_{\mathrm{g}}$. Thus the FES is not necessarily a local optimum for each agent with respect to the set $\Omega$. To determine whether it is locally maximizing on $\Omega_{\mathrm{g}}$, the FES is computed for the facet projection set $\Omega_{\mathrm{g}}$. That is, $\Omega_{\mathrm{g}}$ is assigned as the next set to be searched, and Step 2 is repeated.

Step 3: Check for global optimality. The FES is a local optimum for each agent on the set $\Omega$. For each agent, check whether there is a point on any other feasible facet that results in a higher utility level than the utility level at the FES, holding every other agent's first-period decision variable fixed. If there is a point on another facet at which some agent can obtain higher utility, the candidate solution is not globally maximizing on $\Omega$ for every agent. In this case, select another facet projection set and repeat Step 2. If there is not a point on another facet for which some agent can obtain greater utility, the candidate solution is globally maximizing for all agents over the set $\Omega$, and the FES is therefore an SPE solution.

\subsection{Discussion of the Algorithm}

The number of facets for a two-period SPE problem is $2^{(n+c)}$ where $n$ is the number of period-two variables required to be non-negative, and $\mathrm{c}$ is the number of constraints included in the period-two optimization problems of all agents (excluding non-negativity constraints). This follows from the fact that for every non-negative period-two variable and constraint, there exists a complementarity condition in the KKT conditions for the period-two equilibrium problem. The number of facets is independent of the number of period-one constraints. For the case of no period-two constraints and unrestricted period-two variables $(n=0$ and $c=0)$ there is only one facet. For the numerical example, $\mathrm{n}=2$ and $\mathrm{c}=2$ for a total of 16 facets.

Although the number of facets to be searched is finite, the number increases exponentially according to the number of constraints included in the period-two problem. As a practical matter, the 
number of facets that needs to be searched may be far less than the maximum number of facets. The search for an SPE solution is conducted only on the feasible facets. In the numerical example, the number of facet equilibrium problems that need to be solved is significantly less than the number of facets. Additional aspects of applying the algorithrn are discussed below.

3.2.1 Step 1: Find initial facet. The starting facet can be determined in a number of ways. It is relatively easy to solve an open-loop version of the equilibrium problem (Definition 2) assuming an open-loop solution exists. Any open-loop solution will be on a feasible facet. Therefore, one possibility is to start with the feasible facet that contains the open-loop solution. Alternatively, any facet could be selected initially and a Phase I feasibility problem, as in linear programming, could be solved to determine whether a feasible point existed on the facet. The Phase I problem constraints would consist of the equations and inequalities, which define the facet, augmented by infeasibility variables. If a facet was round to be infeasible, another facet would be tested for feasibility. Since the number of facets is finite, this procedure would either find a feasible facet or conclude that no feasible facet exists and therefore no SPE exists.

\subsubsection{Step 2: Solve facet equilibrium problem}

The solution approach to solving the facet equilibrium problem is based on a bargaining process among the agents:

Step 2.1: Stan with an initial guess $\left(\mathrm{x}_{\mathrm{il}}, \mathrm{x}_{\mathrm{k}:}\right)$ on an initial feasible facet projection set $\Omega_{\mathrm{f}}$.

Step 2.2: Compute $T_{\mathfrak{k}}\left(x_{k 1}\right)$ and $T_{k}\left(x_{i 1}\right)$ on $\Omega_{f}$.

Step 2.3: Compare the previous guess $\left(x_{i 1}, x_{k 1}\right)$ with the computed result $\left[T_{6}\left(x_{k 1}\right), T_{a x}\left(x_{i 1}\right)\right]$ :

Is $\left|\left(x_{i 1}, x_{k 1}\right)-\left[T_{f i}\left(x_{k 1}\right), T_{k}\left(x_{i 1}\right)\right]\right|<$ tolerance?

If yes: The current guess is an FES.

Else: Assign a new guess as follows: 


$$
\begin{aligned}
& x_{i 1} \leftarrow x_{i 1}+\omega\left[T_{i 1}\left(x_{k 1}\right)-x_{i 1}\right] \\
& x_{k 1} \leftarrow x_{k 1}+\omega\left[T_{k x}\left(x_{i 1}\right)-x_{k 1}\right]
\end{aligned}
$$

where $\omega$ is the result of a standard iterative approximation algorithm.

\section{Repeat from Step 2.2.}

In the following situation a choice must be made as part of Step 2. Suppose an FES for the set $\Omega_{\uparrow}$ occurs on the boundary of $\Omega_{\uparrow}$ and the FES is contained in more than one adjacent feasible facet projection set that has not previously been searched for an FES (Figure 5, Case 3). This situation was commonly encountered in solving the numerical example. For example, the FES on $\left(\begin{array}{llll}1 & 1 & 2 & 1\end{array}\right)$ is also

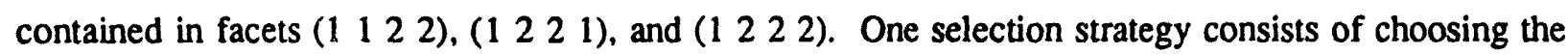
facet that differs from the current facet by the least number of indices starting from the left. In the example given above, this facet selection rule leads to selecting facet $\left(\begin{array}{llll}1 & 2 & 2 & 1\end{array}\right)$ to search next. The use of this rule was found to eliminate cycles in the facet selection that were encountered using other facet selection rules. This selection rule is similar to Bland's pivoting rule for selecting a new basis at a degenerate feasible solution in linear programming (Bland, 1977). An interesting area of future research would be to show that a Bland-type rule or a rule similar to that used in Lemke's Algorithm for selecting almost-complementary solutions (Lemke, 1965) cannot result in a cycle of facets. A variant of Lemke's rule may also have potential application here because an agent's period-one optimization problem consists of an optimization problem on a linear complementarity problem. It would be desirable to show that a facet selection rule necessarily either results in an FES that is locally maximizing on $\Omega$ or concludes that no such solution exists. In this case, no SPE solution exists.

This algorithm could fail in Step 2 if either (1) the FESs had been computed for all facets adjacent to $\Omega_{\mathrm{f}}$ and none was found to be an SPE solution or (2) a locally maximizing FES was found 
to not be globally maximizing for each agent. The problem is in choosing which facet projection set to search next. If all feasible facets have not been searched, then one strategy is to arbitrarily select one of the unsearched facets as the next $\Omega_{\uparrow}$ and proceed to Step 2 . If all feasible facets have been searched unsuccessfully, then two situations are possible: (1) if it is known that a unique FES exists on every feasible facet, then it can be concluded that an SPE solution does not exist, or (2) if it is not known whether FESs are unique, then nothing can be concluded about the existence of an SPE solution. In the second case, the strategy is to search the feasible facets for alternative FESs. Whether this case is likely or even possible to arise is unknown, in that neither of these problems was encountered with the small number of sample problems that were solved as part of this research.

3.2.3 Step 3: Checking for Global Optimality: Step 3 of the algorithm is based on the fact that any SPE solution is by definition globally maximizing for all agents on the set $\Omega$. A test for the global optimality of a solution on facet projection $\Omega_{\mathrm{f}}$ with respect to all agents' utility maximization problems could be conducted as follows. For agent $i$, hold $x_{k 1}$ fixed and solve $P_{g i}\left(x_{k 1}\right)$ for all $g \in F$, $g \neq f$. If the FES on $\Omega_{\mathrm{f}}$ maximizes utility over all facets, the FES is globally maximizing for agent $i$. Repeat the process for agent $k$ while holding $x_{i l}$ fixed. If the FES is globally maximizing for both agents, declare the FES to be an SPE solution. If the local maximizing solution is found not to be globally maximizing for some agent on a particular facet, select that facet as the next facet to search.

\subsection{Illustrátive Results}

The FESs that were computed for the numerical example are shown in Table I and Figure 6. Note from Figure 6 that all FESs are on facet boundaries, with the exception of the SPE solution. In the case where an FES occurs on the boundary of a facet and it is contained in one other facet, the following facet selection rule was successful at eventually leading to an SPE solution: the next facet along a path is selected from those that have a common boundary with the FES on the current facet. 
However, the FESs for several facets were found to be contained in more than one other facet. In this case, the next facet to search needed to be selected from the alternatives. For example, the FES on

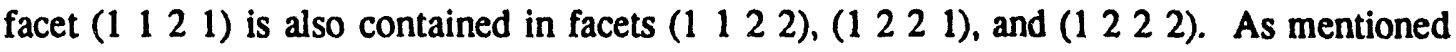
previously, the following rule was found to eliminate cycles in the facet selection process: the next facet selected along a path is the facet that differs from the current facet by the least number of indices starting from the left. For example, for facet $\left(\begin{array}{llll}1 & 1 & 2 & 2\end{array}\right)$ the facet selection rule leads to the selection of facet $\left(\begin{array}{llll}1 & 2 & 2 & 1\end{array}\right)$ as the next facet to search.

Figure 7 shows for each facet which unique facet would be selected as the next to be searched

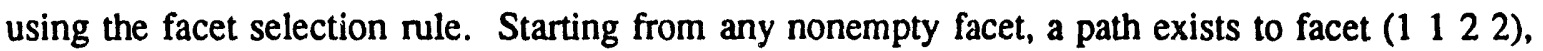
where the SPE solution is located. Note that the FES on $\left(\begin{array}{llll}1 & 1 & 2 & 2\end{array}\right)$ is strictly interior to $\Omega_{1122}$ (Figure 6), and no other facet shares a common boundary with it.

Figure 8 shows the path generated by the algorithm for the starting facet $\left(\begin{array}{llll}1 & 1 & 2 & 1\end{array}\right)$. The starting point for the facet equilibrium algorithm on facet $\left(\begin{array}{llll}1 & 1 & 2 & 1\end{array}\right)$ was obtained by solving a Phase 1

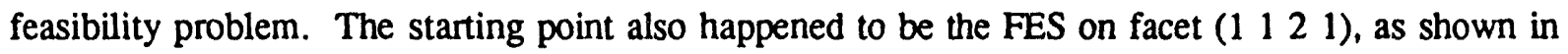
Figure 8. The path was generated by solving the facet equilibrium problems on the successive facets

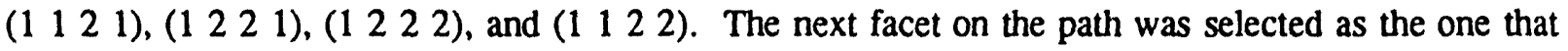
shared a common boundary with the computed FES. A total of 55 iterations were required to go from the starting point on the initial facet to the SPE solution. An iteration consists of solving agent i's period-one maximization problem for $x_{k 1}$ fixed and agent $k$ 's period-one maximization problem for $x_{i 1}$ fixed.

Figure 9 shows the check for global optimality of the candidate SPE solution for both agents' utility maximization problems. Holding $x_{k 1}=5.33615$, the optimal $x_{i 1}$ values for agent $\mathrm{i}$ were computed on each feasible facet. The feasible facets and optimal solutions for agent $i$ are as follows: 


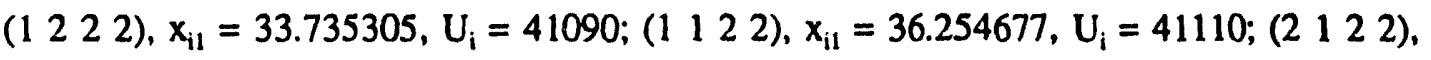
$x_{i 1}=96.213446, U_{i}=30387 ;$ and $\left(\begin{array}{llll}2 & 1 & 1 & 2\end{array}\right), x_{i 1}=97.0, U_{i}=30101$. Thus, the solution $x_{i 1}$ $=36.254677$ is globally optimal for agent $i$ over all feasible facets given $x_{k 1}=5.33615$. The optimal $x_{k 1}$ values for agent $k$ on each feasible facet are computed similarly for fixed $x_{i 1}=36.254677$. The

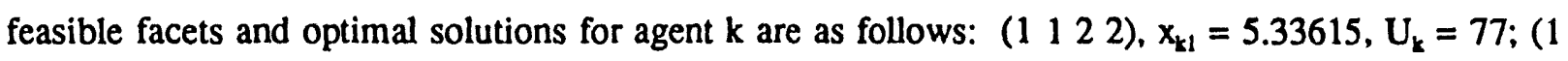
22 2), $x_{k 1}=8.935257, U_{k}=46$; and $\left(\begin{array}{llll}1 & 2 & 2 & 1\end{array}\right), x_{k 1}=52, U_{k}=-5330$. Based on these results, we conclude that the solution $x_{k 1}=5.33615$ is globally optimal for agent $k$ over all feasible facets given $x_{i 1}=36.254677$. This establishes that $\left(x_{i 1}, x_{k 1}, x_{i 2}, x_{k 2}\right)=(36.254677,5.33615,45.42333,0.763438)$ is an SPE solution.

The algorithm could fail if the FES on a facet was not unique. In this case the computed FES could depend on the starting point used for the facet equilibrium calculation. However, the primary candidate for an SPE solution is a strictly interior FES on any facet because any strictly interior FES must satisfy a set of linear equations that represent the first-order conditions for a maximization problem, the solution of which is known to exist.

\section{SUMMARY AND CONCLUSIONS}

In this paper we have described a systematic approach to solving a two-agent, two-period subgame perfect equilibrium formulation under assumptions on the agent objective functions and constraint sets. Specifically, these assumptions are as follows:

1) The KKT conditions for the period-two equilibrium problem are necessary and sufficient.

2) For each $x_{1}$ for which there exists a solution to the period-two equilibrium problem, that solution is unique.

3) $\Lambda$ is a compact set.

4) The Mangasarian-Fromovitz (or Slater given the assumptions regarding functional form) 
constraint qualification holds for each period-one agent optimization problem.

5) For any feasible $\left(x_{i 1}, x_{k 1}\right)$, there exists a unique solution in $\left(x_{i 1}, x_{k 1}\right)$ to the period-one agent optimization problems.

Showing assumptions (1) and (2) to be true for a particular problem is facilitated if there exists a single equivalent optimization problem for the period-two equilibrium problem, as is often the case. Showing that (3) holds is a matter of having a closed and bounded constraint region. Showing that (4) and (5) are satisfied consists of proving properties of solutions to the period-one agent optimization problems. For example, $\mathrm{U}$ quadratic and $\mathrm{G}$ linear reduces the Mangasarian-Fromovitz cunstraint qualification to the Slater constraint qualification.

All results presented here for the two-agent, two-time-period case just discussed hold for any finite number of agents, as can be seen in the definitions and theorems by replacing the production levels attributable to agent $k$ by the sum of production levels attributable to all other agents $k \neq i$ within a time period. For example, take $\sum_{k=1, \alpha ; k x i} x_{k 1}$, rather than $x_{k 1}$, as a fixed value in the period-one optimization problem. Regarding the number of time periods, the structure of the SPE problem is such that the number of facets for a multi-year formulation equals the number of facets in the twoperiod problem after appropriate reductions are made to the multi-year formulation (Macal, 1989). Although the problem size increases in terms of the number of variables as the numbers of agents and periods increases, the number of facets depends solely on the number of constraints. Therefore, the solution approach illustrated in this paper can be used for arbitrary finite numbers of time periods and agents to tile the feasible space of agent optimization problems.

Several extensions to this research are apparent, such as (1) relaxing the compactness assumption on the original constraint set $\Lambda$ and applying an alternative fixed point theorem for unbounded regions, such as the Leray-Schauder Theorem, and (2) investigating altemative facet selection rules. 


\section{ACKNOWLEDGMENTS}

This research was partially supported by the U.S. Department of Energy under contract W-31-109Eng-38 and by the National Science Foundation under grant ECS-8413182.

\section{REFERENCES}

Bard, J.F. and J.E. Falk. 1982. An Explicit Solution to the Multi-Level Programming Problem, Computers and Operations Research, 9(1):77-100.

Bland, R.G. 1977. New Finite Pivoting Rules for the Simplex Method, Mathematics of Operations Research, 2:103-107.

Brouwer, L.E. 1910. Uber eineindentige, stetige Transformationen von Flachen in Sich, Math. Ann., 67:176-180.

Fiacco, A.V. 1983. Introduction to Sensitivity and Stability Analysis in Nonlinear Programming, New York: Academic Press.

Harker, P.T. 1984. A Variational Inequality Approach for the Determination of Oligopolistic Market Equilibria, Mathematical Programming, 30:105-111.

Harker, P.T. 1986. Altemative Models of Spatial Competition, Operations Research, 34(3):410-425, May-June.

Lemke, C.E. 1965. Bimatrix Equilibrium Points and Mathematical Programming, Management Science, 11:681-689.

Macal, C. 1989. A Mathematical Programming Approach to the Formulation and Solution of Subgame

Perfect Equilibrium Problems, Ph.D. dissertation, Northwestem University, Evanston, Ill, available from University Microfilms Int'l, pub. 8913995, vol. 50, issue 4b, Ann Artor, MI.

Macal, C. and A.P. Hurter. 1991. Formulation of Subgame Perfect Equilibrium Problems as 
Mathematical Programs, working paper, November 6.

Mangasarian, O.L., and S. Fromovitz. 1967. The Fritz John Necessary Optimality Conditions in the Presence of Equality and Inequality Constraints, J. Math. Anal. Appl., 17:37-47.

Murphy, F.H., H.D. Sherali, and A.L Soyster. 1982. A Mathematical Programming Approach for Determining Oligopolistic Market Equilibrium, Mathematical Programming, 24:92-106.

Nash, J. 1951. Noncooperative Games, Annals of Mathematics, 54(2):286-295, Sept.

Salant, S.W. 1982. Imperfect Competition in the International Energy Market: A Computerized NashCoumot Model, Operations Research, 30(2):252-280.

Selten, R. 1965. Spieltheoretische Behandlung eines Olihopolmodels mit Nachfragetragheit, Zeitschrift fur die gesamte Staats wissenschaft, 121:301-324 and 667-689.

Selten, R. 1973. A Simple Model of Irnperfect Competition, Where 4 Are Few and 6 Are Many, International Journal of Game Theory, 2:141-201.

Selten, R. 1975. Reexamination of the Perfectness Concept for Equilibrium Points in Extensive Games, Intemational Journal of Game Theory, 4:25-55.

Slater, M. 1950. Lagrange Multipliers Revisited: A Contribution to Nonlinear Programming, Cowles Commission Discussion Paper, Math. 403, Yale Univ., New Haven, Conn. 
Table I Facet Equilibrium Solutions

\begin{tabular}{|c|c|c|c|c|c|c|}
\hline $\begin{array}{c}\text { Facet } \\
\text { Index }\end{array}$ & $x_{i 1}$ & $x_{k 1}$ & $x_{i 2}$ & $x_{12}$ & $\theta_{i}$ & $\theta_{k}$ \\
\hline 1111 & 95 & 41 & 2 & 11 & 0 & 0 \\
\hline 1112 & 97 & 25 & 0 & 15 & 0 & 0 \\
\hline 1121 & 66.4 & 52 & 24 & 0 & 0 & 0 \\
\hline $1122^{2}$ & 36.2547 & 5.3361 & 45.4233 & 0.7634 & 0 & 0 \\
\hline 1211 & 89.5 & 52 & 7.5 & 0 & 0 & 33 \\
\hline 1221 & 26.4999 & 52 & 52.5 & 0 & 0 & 0 \\
\hline 1222 & 35.5550 & 7.9357 & 46.0321 & 0 & 0 & 0 \\
\hline 2111 & 97 & 40 & 0 & 12 & 6 & 0 \\
\hline 2112 & 97 & 0 & 0 & 20 & 0 & 0 \\
\hline 2122 & 96 & 0 & 0 & 20 & 0 & 0 \\
\hline 2211 & 97 & 52 & 0 & 0 & 30 & 48 \\
\hline
\end{tabular}

${ }^{1}$ Empty facets $1212,2121,2212,2221$, and 2222 are not included.

${ }^{2}$ Denotes subgame perfect equilibrium solution. 


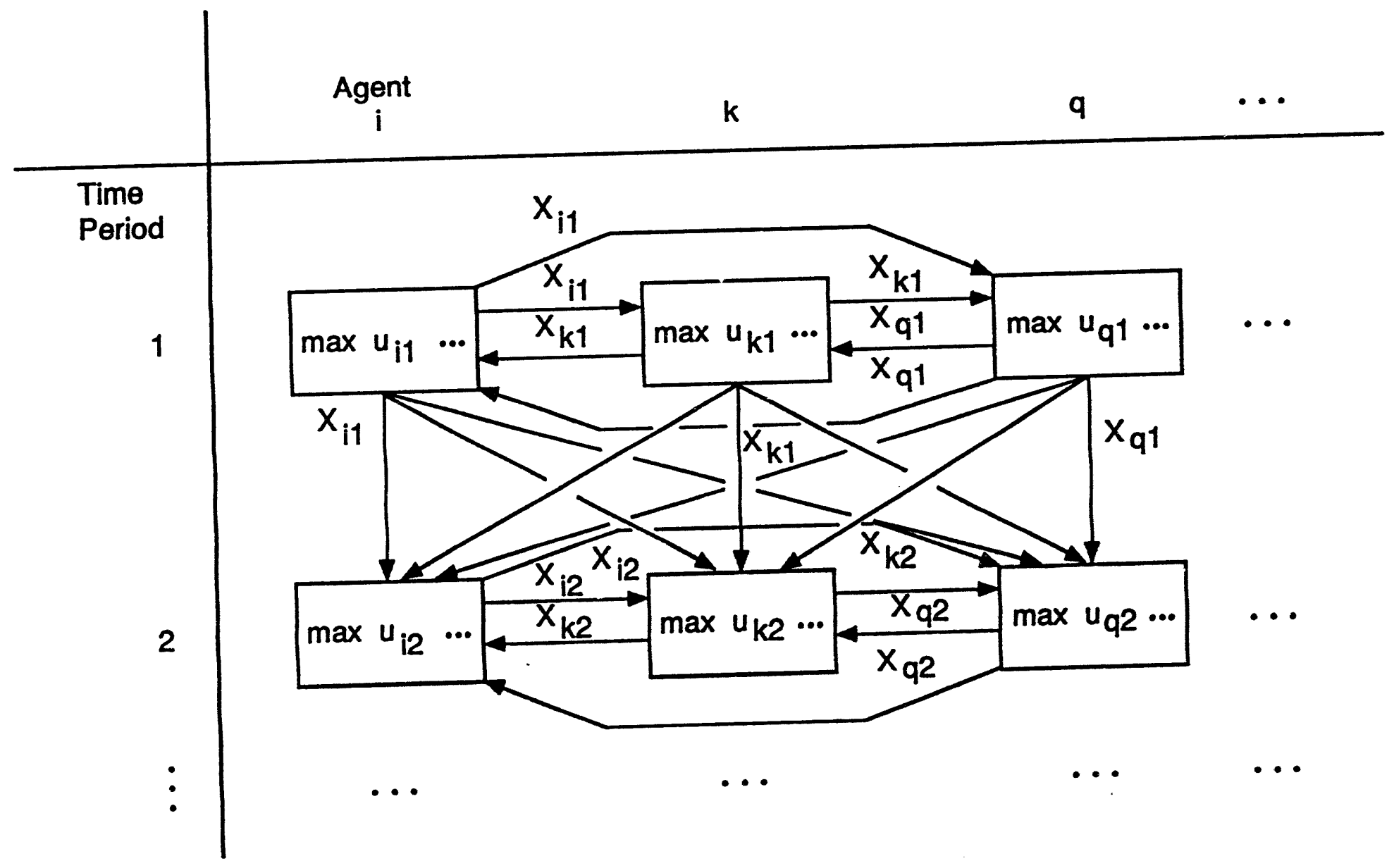

Figure 1 Matrix of Optimization Problems Comprising the Subgame Perfect Equilibrium Problem 


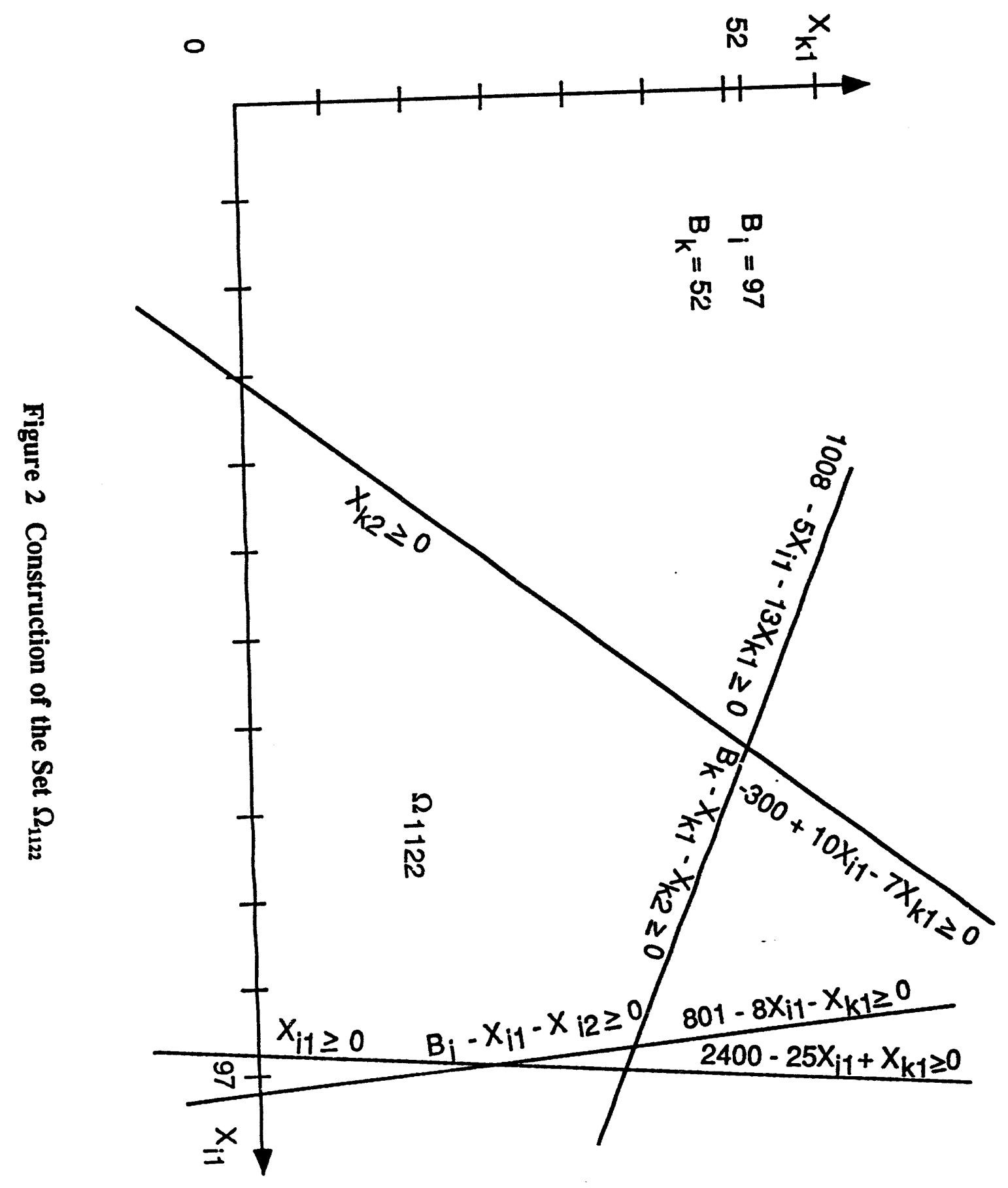




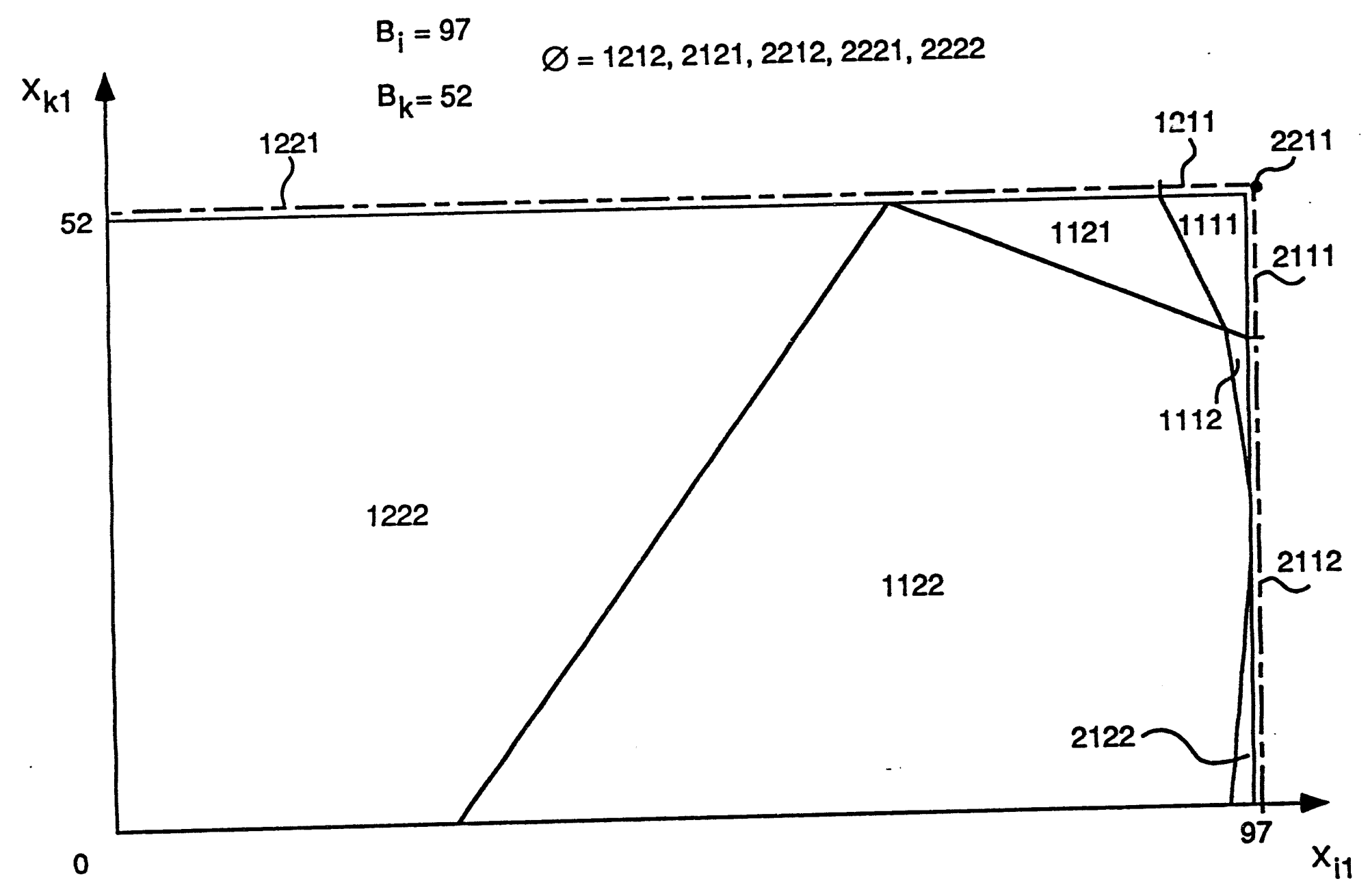

Figure 3 Facet Sets 


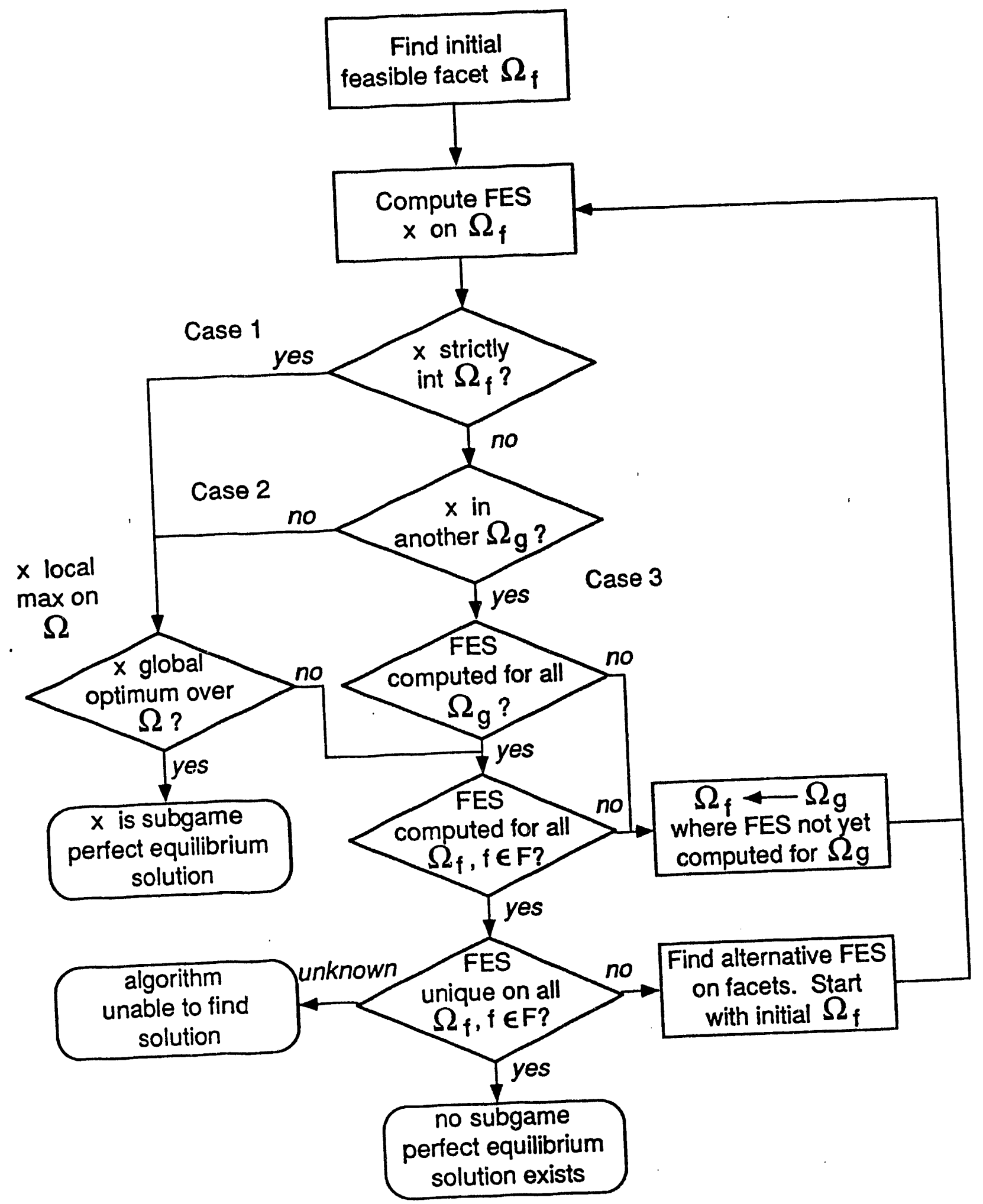

Figure 4 Algorithm Flowchart 
Case 1:

FES $\in$ int $\Omega_{f}$

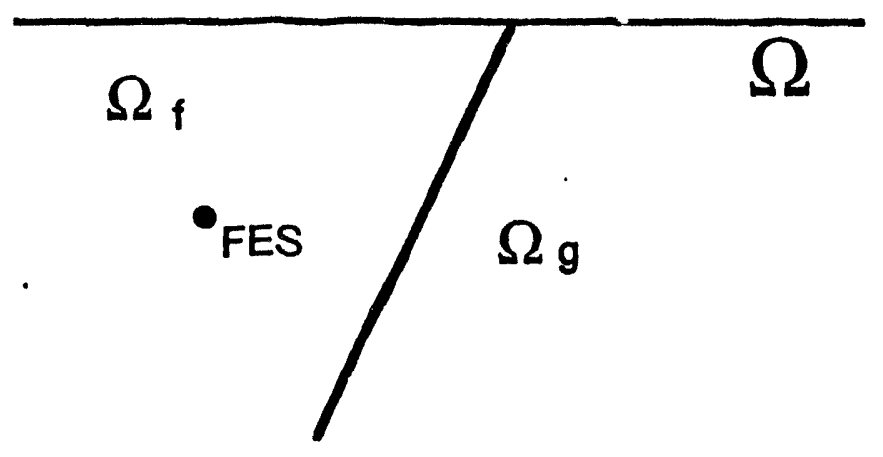

Case 2:

FES $\in \partial \Omega_{f}$ FES $\notin \Omega \mathrm{g}$

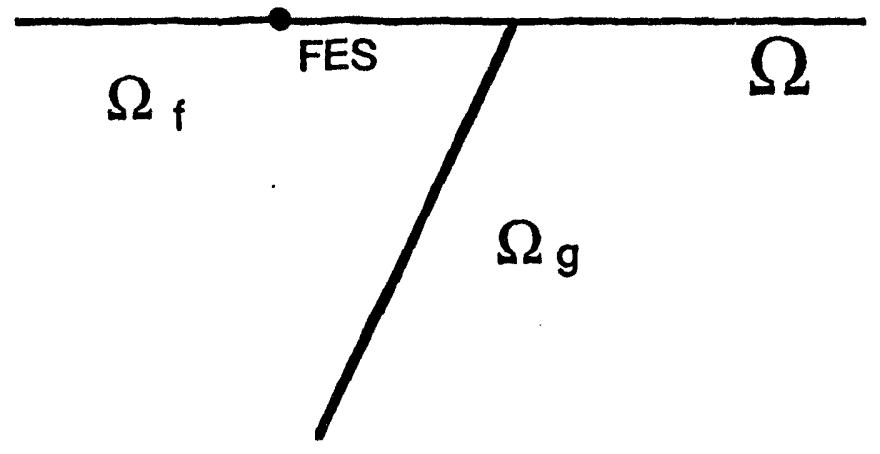

Case 3:

FES $\in \partial \Omega_{f}$ FES $\in \Omega_{\mathrm{g}}$

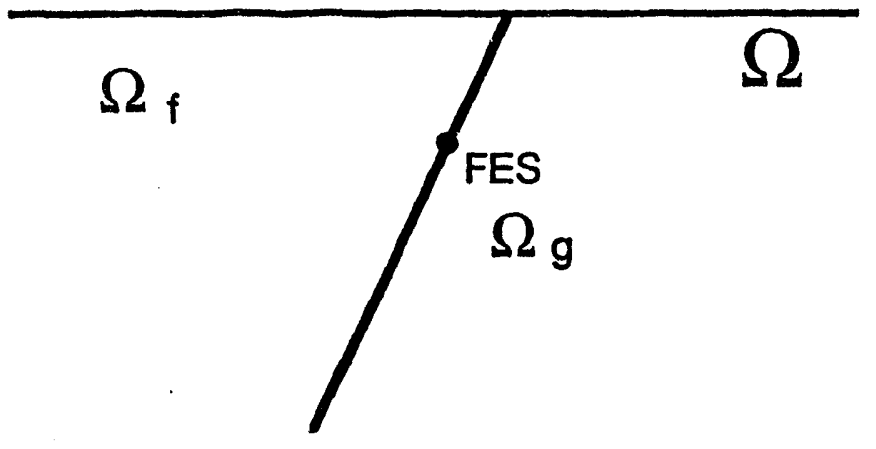

Figure 5 Cases for Facet Equilibrium Solutions

FES indicates facet equilibrium solution 


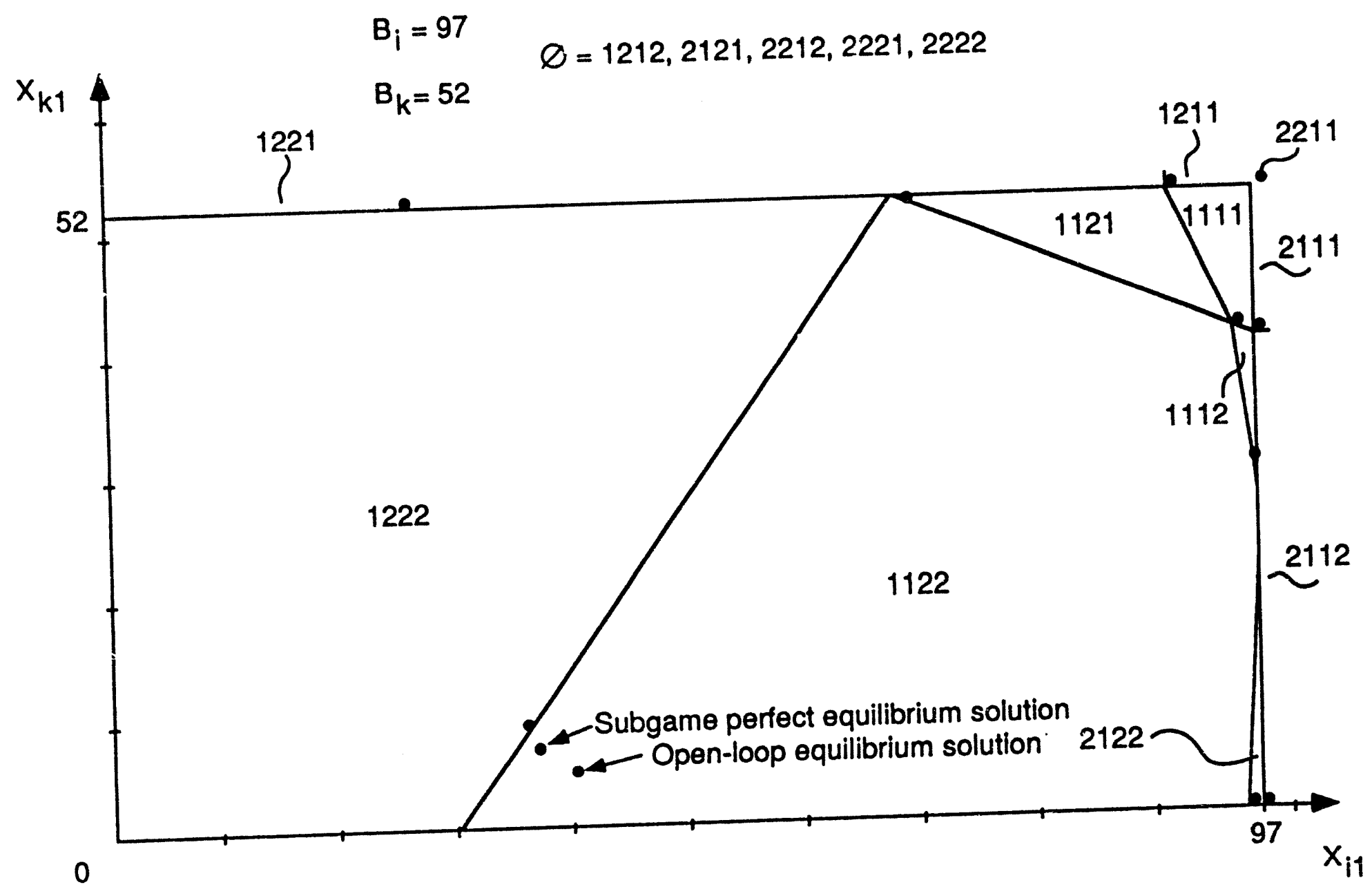

Figure 6 Facet Equilibrium Solutions 


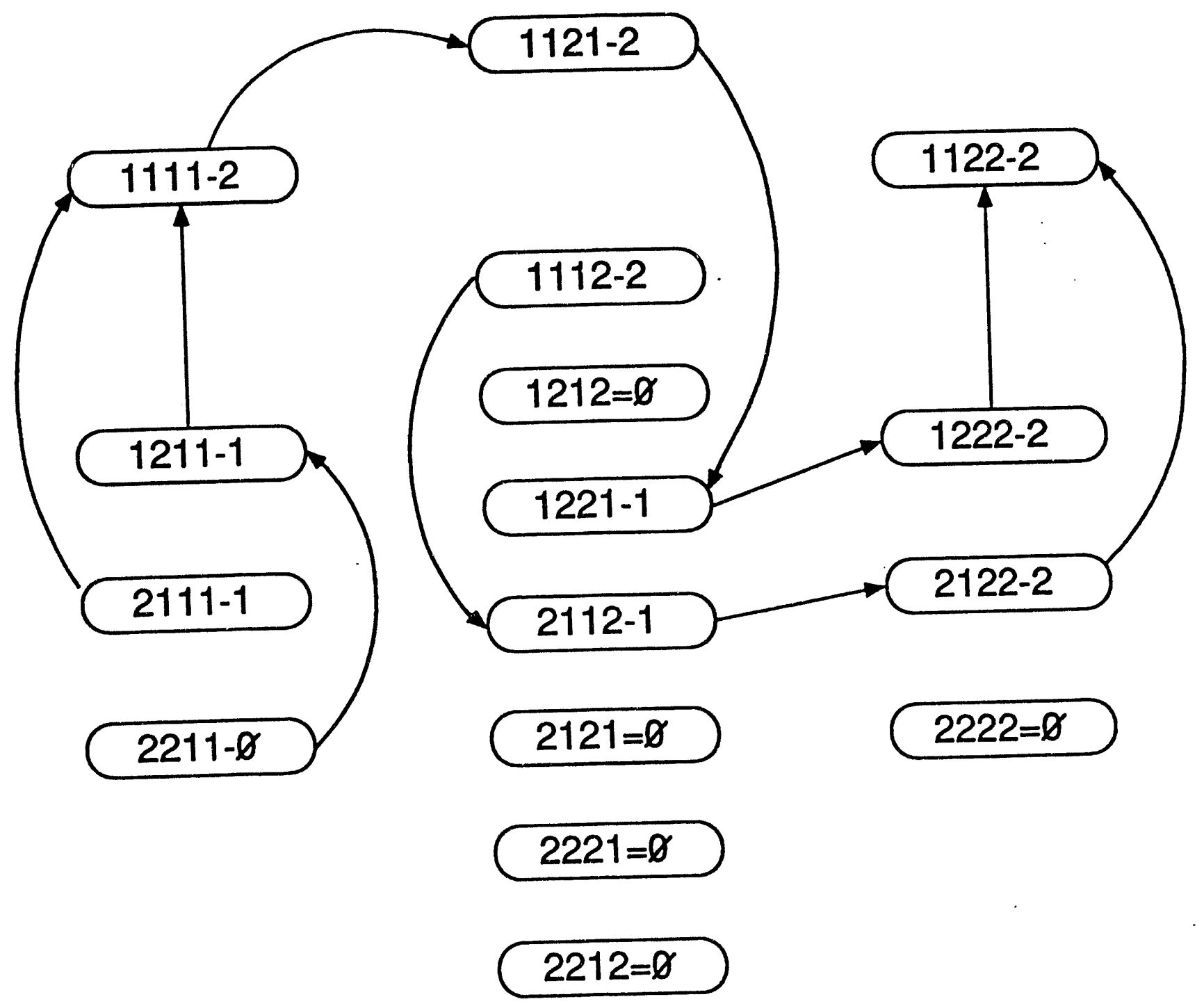

Figure 7 Pointers of Facet Equilibrium Solutions to Other Facets $\varnothing$ indicates empty facet.

Facet index-n indicates that $\Omega_{\mathrm{f}}$ is of dimension $n$. 


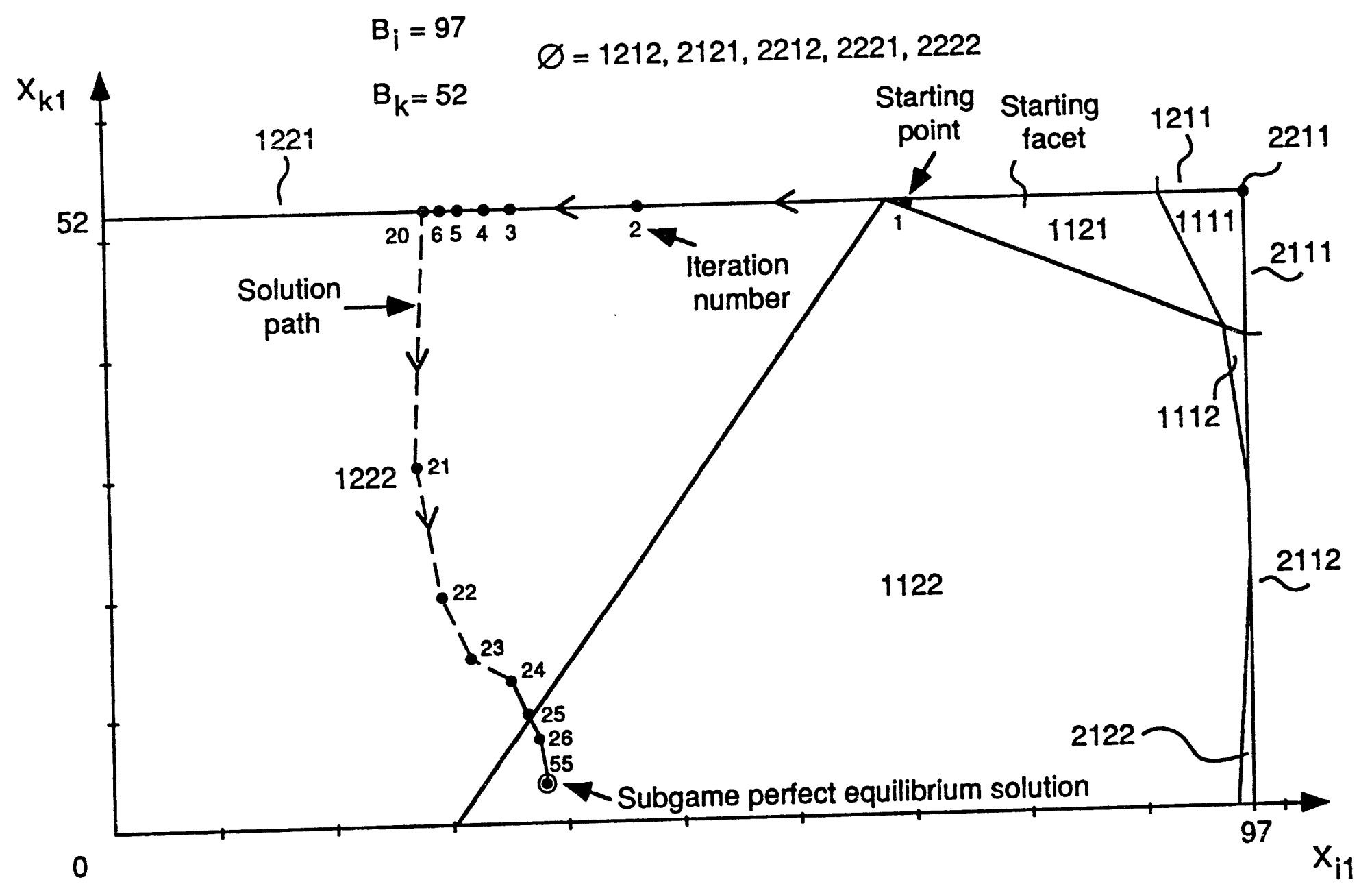

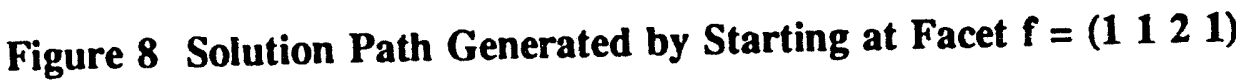






Figure 9 Check for Global Optimality of the Subgame Perfect Equilibrium Solution Over the Set $\Omega$ 

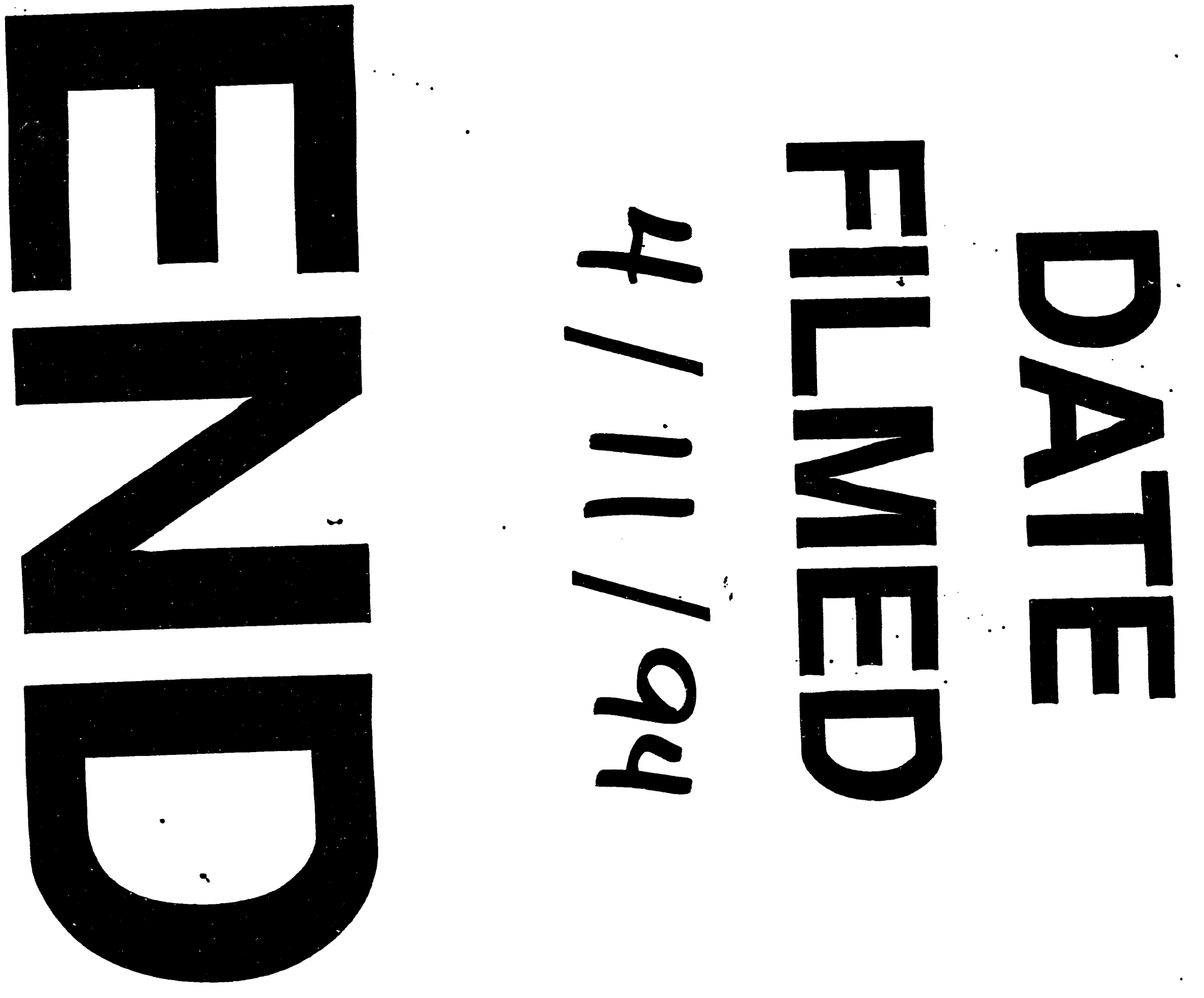


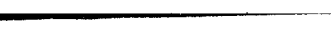

